\title{
The Diversity of Type II Supernova vs. The Similarity in Their Progenitors
}

\author{
S. Valenti ${ }^{\star}$, D. A. Howell ${ }^{2,3}$, M. D. Stritzinger ${ }^{4}$, M. L. Graham ${ }^{5}$, G. Hosseinzadeh ${ }^{2,3}$, \\ I. Arcavi ${ }^{2,6}$, L. Bildsten ${ }^{6}$, A. Jerkstrand ${ }^{7}$, C. McCully ${ }^{2,3}$, A. Pastorello ${ }^{8}$, A. L. Piro ${ }^{9}$, \\ D. Sand ${ }^{10}$, S. J. Smartt ${ }^{7}$, G. Terreran ${ }^{7,8}$, C. Baltay ${ }^{11}$, S. Benetti ${ }^{8}$, P. Brown $^{12}$, \\ A. V. Filippenko ${ }^{5}$, M. Fraser ${ }^{13}$, D. Rabinowitz ${ }^{11}$, M. Sullivan ${ }^{14}$, F. Yuan ${ }^{15,16}$ \\ 1 Department of Physics, University of California, Davis, CA 95616, USA \\ 2 Las Cumbres Observatory Global Telescope Network, 6740 Cortona Dr., Suite 102, Goleta, CA 93117, USA \\ 3 Department of Physics, University of California, Santa Barbara, Broida Hall, Mail Code 9530, Santa Barbara, CA 93106-9530, USA \\ 4 Department of Physics and Astronomy, Aarhus University, Ny Munkegade 120, DK-8000 Aarhus C, Denmark \\ 5 Department of Astronomy, University of California, Berkeley, CA 94720-3411, USA \\ 6 Kavli Institute for Theoretical Physics, Kohn Hall, University of California, Santa Barbara, CA 93106-4030, USA \\ 7 Astrophysics Research Centre, School of Mathematics and Physics, Queens University Belfast, Belfast BT7 1NN, UK \\ 8 INAF Osservatorio Astronomico di Padova, Vicolo dell'Osservatorio 5, 35122 Padova, Italy \\ 9 Carnegie Observatories, 813 Santa Barbara Street, Pasadena, CA 91101, USA \\ 10 Physics Department, Texas Tech University, Lubbock, TX, 79409, USA \\ 11 Department of Physics, Yale University, New Haven, CT 06520-8120, USA \\ 12 George P. and Cynthia Woods Mitchell Institute for Fundamental Physics \& Astronomy, \\ Texas A. \&S M. University, Department of Physics and Astronomy, 4242 TAMU, College Station, TX 7r843, USA \\ 13 Institute of Astronomy, University of Cambridge, Madingley Road, Cambridge, CB3 OHA, UK \\ 14 School of Physics and Astronomy, University of Southampton, Southampton, SO17 1BJ, UK \\ 15 Research School of Astronomy and Astrophysics, Australian National University, Canberra, ACT 2611, Australia \\ ${ }^{16}$ ARC Centre of Excellence for All-Sky Astrophysics (CAASTRO)
}

Accepted ....; Received ...; in original form ....

\begin{abstract}
High-quality collections of Type II supernova (SN) light curves are scarce because they evolve for hundreds of days, making follow-up observations time consuming and often extending over multiple observing seasons. In light of these difficulties, the diversity of SNe II is not fully understood. Here we present ultraviolet and optical photometry of 12 SNe II monitored by the Las Cumbres Observatory Global Telescope Network (LCOGT) during 2013-2014, and compare them with previously studied SNe having well-sampled light curves. We explore SN II diversity by searching for correlations between the slope of the linear light-curve decay after maximum light (historically used to divide SNe II into IIL and IIP) and other measured physical properties. While SNe IIL are found to be on average more luminous than SNe IIP, SNe IIL do not appear to synthesize more ${ }^{56} \mathrm{Ni}$ than SNe IIP. Finally, optical nebular spectra obtained for several SNe in our sample are found to be consistent with models of red supergiant progenitors in the $12-16 \mathrm{M}_{\odot}$ range. Consequently, SNe IIL appear not to account for the deficit of massive red supergiants as SN II progenitors.
\end{abstract}

Key words: supernovae: general - supernovae: individual: SN 2013bu, SN 2013fs, SN 2014cy, SN 2013ej, ASASSN-14ha, ASASSN-14gm, ASASSN-14dq, SN 2013ab, SN 2013by, SN 2014G, LSQ13dpa, LSQ14gv, SN 2014G, SN 2013ab, SN 2015W.

\section{INTRODUCTION}

* e-mail: stfn.valenti@gmail.com

The majority of core-collapse supernovae (SNe) are associated with the deaths of hydrogen-rich massive stars

(C) 0000 The Authors 
with zero-age-main-sequence masses $M_{\mathrm{ZAMS}}>8 \mathrm{M}_{\odot}$. The most common hydrogen-rich Type II SNe exhibit a 100 day plateau in their light curves (SNe IIP), and the physics behind their emission is well understood. In particular, the energy source powering the plateau phase is the energy deposited by the shock wave soon after the explosion. The recombination process of the ionized hydrogen is responsible for the photosphere moving inward and allowing the stored energy to be radiated during this phase (Popov 1993; Kasen \& Woosley 2009; Dessart \& Hillier 2010; Bersten 2013). The recombination wave travels through the hydrogen-rich ejecta as the SN expands homologously and cools (Falk \& Arnett 1973; Chevalier 1976).

However, a fraction of SNe II have a linear decline in their light curves after a rapid rise to maximum light (SNe IIL; Barbon, Ciatti, \& Rosino 1979; Filippenko 1997). After either the plateau or linear decline phase in SNe II there is a subsequent rapid drop, usually around 100-140 days after explosion for SNe IIP and 80-100 days for SNe IIL (Valenti et al. 2015). This difference may be due to a smaller ejecta mass in SNe IIL. Blinnikov \& Bartunov (1993) argued that the fast light-curve decline of SNe IIL is the result of both the small amount $\left(1-2 \mathrm{M}_{\odot}\right)$ of hydrogen envelope in a progenitor of relatively larger radius (a few $1000 \mathrm{R}_{\odot}$ ). These values can be compared with the progenitors of SNe IIP, which are red supergiants (RSGs) that span radii of $10-1600 R_{\odot}$ (Levesque et al. 2005) at the moment of explosion and should have an ejected mass of $\sim 6-10$ $\mathrm{M}_{\odot}$. Recent hydrodynamical and radiative-transfer calculations have also confirmed that a smaller ejected mass may produce a short plateau, but cannot reproduce the luminosity of SNe IIL (Morozova et al. 2015; Moriya et al. 2015). A larger radius, a different hydrogen distribution, or extra energy sources may play an important role in shaping the light curves of SNe IIL.

RSGs have been definitively identified as the progenitors of SNe IIP (e.g., Maund, Smartt, \& Danziger 2005; Fraser et al. 2011; Van Dyk et al. 2012), with masses estimated from the measured luminosities of the progenitor stars to be in the range $8-17 \mathrm{M}_{\odot}($ Smartt 2009). The fact that more-massive RSG progenitors have not been identified has been highlighted as significant and termed the "red supergiant problem" (Smartt et al. 2009).

The separation, if any, between SNe IIP and IIL is an open issue. Some authors suggest that they form a continuous distribution (Patat et al. 1994; Anderson et al. 2014; Sanders et al. 2015; Valenti et al. 2015), while others favour their separation into distinct classes (Arcavi et al. 2012; Faran et al. 2014a,b). If one of the keys to differentiating SNe IIP from IIL is the amount of hydrogen in the envelope, a clear dichotomy may suggest the presence of a specific, unknown ingredient in the evolution of massive stars that sometimes strips them of discrete (large for SNe IIL, small for SNe IIP) amounts of hydrogen. Despite an increasing number of SNe II with published light curves and the growing evidence that SNe II form a continuum of light-curve properties, it remains unknown whether their progenitors span a range of stellar populations.

In this paper, we present comprehensive optical light curves of 12 SNe II that have been monitored by the Las Cumbras Observatory Global Telescope (LCOGT,
Brown et al. 2013) Network between 2013 and 2014. The LCOGT network of nine $1 \mathrm{~m}$ and two $2 \mathrm{~m}$ robotic telescopes enables photometric coverage with long temporal baselines and fast response times to cover the most timecritical phases of SN evolution. Many of these SNe II have also been followed at ultraviolet (UV) wavelengths with the UVOT camera onboard Swift (Roming et al. 2005). When possible, we will compare our data with those previously published and publicly available (see next section). The main goal of this paper is to focus on generic properties of our sample to gain an expanded understanding of the SN IIP/IIL diversity.

Various authors use different values of the linear decay rate after maximum brightness to separate SNe IIP and SNe IIL. The reader should be aware that in this paper, we will not define a specific decline-rate value to distinguish SNe IIP and SNe IIL; rather, we will use IIP to refer to SNe II with flatter linear decays after maximum light (IIP-like SNe) and IIL to refer to SNe II with faster decline rates after maximum light (IIL-like $S N e$ ). When possible, we will colour-code the sample of objects using the decline rate of the $V$-band light curve $\left(s_{V 50}\right.$; see Section 4 for the definition) to emphasize that we are not dividing SNe II to IIP and IIL a priori.

In Section 2, we present our sample of new SNe as well as $\mathrm{SNe}$ from the literature that are used in the analysis. In Section 3 we present our photometric data, and Section 4 describes the main parameters characterizing them (listed also in the Appendix D). We repeat the analysis in Section 5 using pseudobolometric light curves. In Section 6 we discuss early-time data, focusing on the UV differences between SNe IIP and IIL. We derive in Section 8 the amount of ${ }^{56} \mathrm{Ni}$ produced in SNe II and compare our results with progenitor studies of SNe II (Smartt et al. 2009). In Section 9 we constrain the mass of the progenitor for a few $\mathrm{SNe}$ in our sample using their nebular spectra, and Section 10 summarizes our results.

\section{SUPERNOVA SAMPLE}

Between September 2012 and July 2013, LCOGT deployed eight $1 \mathrm{~m}$ telescopes around the world. Commissioning of the telescopes lasted until March 2014 when the LCOGT network of telescopes become operational (Brown et al. 2013). It was at this time we initiated our programme to obtain detailed follow-up observations of SNe II. To maximize the potential of the LCOGT network to contribute to our understanding of SNe II, we elected to monitor all objects located within $40 \mathrm{Mpc}$ and having constraints on the explosion epoch of within one week or less, or a blue featureless spectrum which is typical of young SNe II. Objects selected for monitoring were discovered by various transient search surveys (see below), and our main criterion for selection was the potential to collect data extending from soon after explosion to at least the end of the plateau phase.

The sample consists of 16 bright SNe II discovered in 2013 and 2014. Data for 12 of the objects are presented here for the first time, while photometry of 4 objects (SN 2013ej, Valenti et al. 2014, Yuan et al., in preparation; SN 2013by, Valenti et al. 2015; SN 2014G, Terreran et al., in preparation; SN 2013ab, Bose et al. 2015) are presented in papers 
Table 1. LCOGT Type II Supernovae

\begin{tabular}{|c|c|c|c|c|c|c|c|c|}
\hline $\mathrm{SN}$ & Discovery & Ref-discovery $^{*}$ & $\begin{array}{c}\text { Classification }^{\top} \\
\text { SN II }\end{array}$ & $\begin{array}{c}\text { Last } \\
\text { nondetection }\end{array}$ & $\begin{array}{c}\text { Photometry } \\
\text { coverage (days) }\end{array}$ & $\mathrm{N}$ points & $\begin{array}{c}\alpha(\mathrm{J} 2000) \\
(\mathrm{h}: \mathrm{m}: \mathrm{s})\end{array}$ & $\begin{array}{c}\delta(\mathrm{J} 2000) \\
\left({ }^{\circ}:^{\prime}:^{\prime \prime}\right)\end{array}$ \\
\hline $2013 \mathrm{fs}$ & 2013 Oct. 07.46 & a & young, aa & 2013 Oct. $5.24, \mathrm{o}$ & 106 & 371 & $23: 19: 44.67$ & $+10: 11: 04.5$ \\
\hline 2013bu & 2013 Apr. 21.76 & $\mathrm{~b}$ & young, bb & 2013 Apr. $12.8, \mathrm{p}$ & 130 & 163 & $22: 37: 05.60$ & $+34: 24: 31.9$ \\
\hline ASASSN-14ha & 2014 Sep. 10.29 & ASASSN, c & young, cc & 2014 Sep. 08.3, c & 180 & 372 & 04:20:01.41 & $-54: 56: 17.0$ \\
\hline ASASSN-14dq & 2014 Jul. 8.48 & ASASSN, d & SN II, dd & 2014 Jun. 27.49, d & 341 & 219 & $21: 57: 59.97$ & $+24: 16: 08.1$ \\
\hline ASASSN-14gm & 2014 Sep. 2.47 & ASASSN, e & young, ee & 2014 Aug. 30.48, e & 318 & 327 & $00: 59: 47.83$ & $-07: 34: 19.3$ \\
\hline $2014 \mathrm{cy}$ & 2014 Aug. 31.0 & $\mathrm{f}$ & SN II , ff & 2013 Aug. 29.3, LOSS & 160 & 162 & $23: 44: 16.03$ & $+10: 46: 12.5$ \\
\hline ASASSN-14kg & 2014 Nov. 17.36 & ASASSN, g & young, gg & 2014 Nov. 11.38, g & 100 & 99 & $01: 44: 38.38$ & $+35: 48: 20.5$ \\
\hline 2013ai & 2013 Mar. 1.66 & $\mathrm{~h}$ & young, hh & - & 83 & 163 & $06: 16: 18.35$ & $-21: 22: 32.5$ \\
\hline $2014 d w$ & 2014 Nov. 6.59 & $\mathrm{i}$ & young, ii & - & 141 & 149 & $11: 10: 48.41$ & $-37: 27: 02.2$ \\
\hline LSQ13dpa & 2013 Dec. 18.28 & LSQ, l & young, ll & 2013 Dec $14.2,1$ & 189 & 179 & 11:01:12.91 & $-05: 50: 52.4$ \\
\hline LSQ14gv & 2014 Jan. 17.3 & LSQ, m & young, $\mathrm{mm}$ & 2014 Jan. 13.17, m & 97 & 113 & $10: 54: 11.71$ & $-15: 01: 30.0$ \\
\hline $2015 \mathrm{~W}$ & 2015 Jan. 12.17 & LOSS, n & SN II, nn & 2014 Dec. $14, \mathrm{n}$ & 119 & 221 & $06: 57: 43.03$ & $+13: 34: 45.7$ \\
\hline
\end{tabular}

(*) a, Nakano et al. (2013); b, Itagaki et al. (2013); c, Kiyota et al. (2014); d, Stanek et al. (2014); e, Holoien et al. (2014); f, Nishimura (2014); g, Nicolas et al. (2014); h, Conseil et al. (2013a); i, Parker (2015); 1, Hsiao et al. (2013); m, Ergon et al. (2014); n, Kim, H.; Zheng (2015).

(†) aa, Childress et al. (2013); bb, Ochner et al. (2013); cc, Arcavi et al. (2014b); dd, Arcavi et al. (2014c); ee, Elias-Rosa et al. (2014); ff, Morrell et al. (2014); gg, Falco et al. (2014); hh, Conseil et al. (2013b); ii, Arcavi et al. (2014a); 1l, Hsiao et al. (2013); mm, Ergon et al. (2014); nn, Tomasella et al.

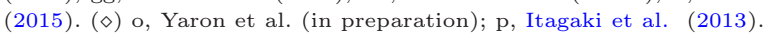

Table 2. Sample of Type II Supernovae

\begin{tabular}{|c|c|c|c|c|c|c|c|c|c|}
\hline Object $^{+}$ & $\begin{array}{l}\text { Distance } \\
\text { modulus }\end{array}$ & $\begin{array}{c}\text { Ref. }^{*} \\
(\mu)\end{array}$ & $\begin{array}{c}\text { Explosior } \\
\text { epoch }\end{array}$ & & $\begin{array}{c}\text { m Ref. }^{*} \\
\text { (explosion) }\end{array}$ & $\begin{array}{c}E(B-V) \\
\text { (host) }\end{array}$ & $\begin{array}{c}\text { Ref. }^{*} \\
\left(E(B-V)_{\text {host }}\right)\end{array}$ & $\begin{array}{l}E(B-V)^{x} \\
\text { M.W. }\end{array}$ & $\begin{array}{l}\text { Host } \\
\text { galaxy }\end{array}$ \\
\hline SN 2013ai & 32.200 .15 & $\mathrm{a}$ & 2456348.00 & 5.0 & $\mathrm{w}$ & 0.150 .08 & $\mathrm{w}$ & 0.08 & NGC 2207 \\
\hline SN 2013bu & 30.790 .08 & b & 2456399.80 & 4.5 & $\mathrm{w}$ & 0.000 .00 & $\mathrm{w}$ & 0.08 & NGC 7331 \\
\hline lsq13dpa & $\begin{array}{lll}35.08 & 0.15\end{array}$ & $\mathrm{a}$ & 2456642.70 & 2.0 & $\mathrm{w}$ & 0.000 .00 & $\mathrm{w}$ & 0.04 & LCSBS 14920 \\
\hline SN 2014cy & 31.870 .15 & a & 2456900.00 & 1.0 & $\mathrm{w}$ & 0.000 .00 & $\mathrm{w}$ & 0.05 & NGC 7742 \\
\hline SN $2014 \mathrm{dw}$ & 32.460 .15 & $\mathrm{a}$ & 2456958.00 & 10.0 & $\mathrm{w}$ & 0.110 .06 & $\mathrm{w}$ & 0.11 & NGC 3568 \\
\hline lsq14gv & 35.150 .15 & a & 2456674.80 & 2.0 & $\mathrm{w}$ & 0.000 .00 & $\mathrm{w}$ & 0.06 & 2MASX J10541092-1501228 \\
\hline ASASSN-14gm & 31.740 .15 & $\mathrm{a}$ & 2456901.00 & 1.5 & $\mathrm{w}$ & 0.000 .00 & $\mathrm{w}$ & 0.10 & NGC 337 \\
\hline ASASSN-14kg & 33.830 .15 & $\mathrm{a}$ & 2456970.00 & 3.0 & $\mathrm{w}$ & 0.250 .12 & $\mathrm{w}$ & 0.04 & CGCG 521-075 \\
\hline ASASSN-14ha & 29.530 .50 & $\mathrm{a}$ & 2456910.50 & 1.5 & $\mathrm{w}$ & 0.000 .00 & $\mathrm{w}$ & 0.01 & NGC 1566 \\
\hline SN 2015W & 33.740 .15 & $\mathrm{a}$ & 2457025.00 & 10.0 & $\mathrm{w}$ & 0.000 .00 & $\mathrm{w}$ & 0.15 & UGC 3617 \\
\hline SN 2013ab & 31.900 .08 & $\mathrm{~d}$ & 2456340.00 & 1.0 & $\mathrm{~d}$ & 0.020 .07 & $\mathrm{~d}$ & 0.02 & $1430+101$ \\
\hline SN 2013by & 30.810 .15 & $\mathrm{a}$ & 2456404.00 & 2.0 & $\mathrm{x}$ & $0.00 \quad 0.00$ & $\mathrm{x}$ & 0.23 & ESO $138-G 010$ \\
\hline SN 2013ej & 29.790 .20 & $\mathrm{c}$ & 2456515.10 & 1.0 & $\mathrm{y}$ & $0.00 \quad 0.00$ & $\mathrm{y}$ & 0.06 & M74 \\
\hline SN $2014 \mathrm{G}$ & 31.900 .15 & $\mathrm{a}$ & 2456668.35 & 1.0 & $\mathrm{w}$ & 0.200 .00 & $\mathrm{kk}$ & 0.01 & NGC 3448 \\
\hline- & - & - & - & & - & - & - & - & - \\
\hline
\end{tabular}

+ Table continues in the online version with objects from literature.

* a: NED , b: Kanbur et al. 2003, c: Fraser et al. 2014, d: Bose et al. 2015, e: Brown et al. 2010, f: Ferrarese et al. 1996, g: Schmidt, Kirshner, \& Eastman 1992, h: Poznanski et al. 2009, i: Jones et al. 2009, j: Leonard et al. 2003, k: Wang et al. 2006, 1: Takats \& Vinko 2006, m: Anderson et al. 2014, n: Takáts \& Vinkó 2012, o: Bose \& Kumar 2014, p: Freedman et al. 2001, q: Takáts et al. 2014, r: Takats et al. 2015, s: Gall et al. 2015, t: Olivares et al. 2010, u: Mould \& Sakai 2008, v: Rest et al. 2014, w: This paper, x: Valenti et al. 2015, z: Benetti et al. 1994, aa: Faran et al. 2014b, bb: Inserra et al. 2013, cc: Taddia et al. 2013, dd: Spiro et al. 2014, ee: Faran et al. 2014a, ff: Pozzo et al. 2006, gg: de Vaucouleurs et al. 1981, hh: Leonard et al. 2002b, jj: Pastorello et al. 2004, kk: Terreran in preparation, 11: Yaron in preparation, mm: Gandhi et al. 2013, nn: Maguire et al. 2010, oo: Pastorello et al. 2009, pp: Inserra et al. 2011, qq: Tomasella et al. 2013, rr: Barbarino et al. 2015, ss: Inserra et al. 2012, tt: Fraser et al. 2011, uu: Gal-Yam et al. 2011, vv: Elias-Rosa et al. 2011, ww: Dessart et al. 2008, xx: Dall'Ora et al. 2014, zz: Poznanski et al. 2015, ab: Elias-Rosa et al. 2010, ac: Zwitter, Munari, \& Moretti 2004, ad: Quimby et al. 2007, ae: Andrews et al. 2011.

cited herein though their data are included in our analysis below.

Information characterizing our SNe II is reported in Table 1, including details concerning the discovery, classification, duration of monitoring, and coordinates. Most of the objects were discovered 1-4 days after explosion and observed for more than 100 days, covering the end of the plateau phase. Unfortunately, the end of the plateau phase was not included for SN 2013ai and ASASSN-14kg as they disappeared into the daytime sky.

Four objects within the sample were discovered by the All-Sky Automated Survey for SuperNovae (ASASSN ${ }^{1}$, Shappee et al. 2014), two by the La Silla Quest Variability Survey $^{2}$ (Baltay et al. 2013), and one by the Lick Observa- tory Supernova Search (LOSS; Li 2000; Filippenko et al. 2001) with the Katzman Automatic Imaging Telescope (KAIT). In addition, five objects were reported on the Transient Objects Confirmation Page (TOCP) of the Central Bureau for Astronomical Telegrams $\left(\mathrm{CBAT}^{3}\right)$. More details are available in Appendix A.

We will compare our sample of $16 \mathrm{SNe}$ II with data of other $\mathrm{SNe}$ II from the literature. We consider all SNe II with dense photometric follow-up observations that are presented by Anderson et al. (2014), Faran et al. (2014a), Faran et al. (2014b), Poznanski et al. (2015), and Spiro et al. (2014), as well as with several recent well-observed SNe II (Tomasella et al. 2013; Dall'Ora et al. 2014; Takáts et al. 2014; Takats et al.

1 www.astronomy.ohio-state.edu/ assassin/index.shtml

2 LSQ, http://hep.yale.edu/lasillaquest

3 CBAT, http://www.cbat.eps.harvard.edu/ 
2015; Gall et al. 2015; Pastorello et al. 2009; Inserra et al. 2013; Leonard et al. 2002a).

We stress that the number of objects taken from the literature and used for comparison in the following sections changes depending on the exact data available for each object. For example, including our sample, $107 \mathrm{SNe}$ II have been observed during the linear decay phase allowing for an estimate of the linear decline rate over 50 days, but only 48 of these objects have $V$-band observations that enable a measurement of the drop at the end of the linear decay. Moreover, at early phases and in the UV the number of objects with good data is even smaller (see Section 6).

\section{OBSERVATIONS}

The LCOGT images were reduced using lcogtsnpipe, a custom pipeline developed by one of us (S.V.). The pipeline computes magnitudes with the point-spread-function (PSF) fitting technique and a low-order polynomial fit removes the host-galaxy background. The template-subtraction technique (using HOTPANTS ${ }^{4}$ ) was used in the case of SN 2013bu, SN 2014cy, SN 2014dw, LSQ14gv, and LSQ13dpa, as in those cases the host-galaxy contamination was particularly relevant. The absolute calibration was performed using APASS ${ }^{5}$, which implies that $B$ and $V$ are calibrated to the Landolt system (Vega), while $g, r$, and $i$ are calibrated to the Sloan system (AB magnitudes). Details about the pipeline can be found in Appendix B. Swift aperture photometry was computed following the prescriptions described by Brown et al. (2009), but using the updated zeropoints of Breeveld et al. (2010). Swift spectra were reduced using uvotpy ${ }^{6}$. LSQ data for LSQ14gv and LSQ13dpa were reduced following the prescriptions described by Firth et al. (2014).

We also obtained six nebular spectra of three of our targets at the Keck and Gemini North Observatories. SNe II are faint during their nebular phase; hence, it was not possible to obtain spectra of the entire sample. These spectra together with nebular spectra from the literature are used in Section 9 to constrain the zero-age-main-sequence mass of the progenitors.

The Keck spectra are of ASASSN-14dq (2015 June 16.47, 2015 Oct. 10.34), ASASSN-14gm (2015 Oct. 10.39), and SN 2015W (2015 Oct. 10.60) with the Low Resolution Imaging Spectrometer (LRIS; Oke et al. 1995). We used the $1.0^{\prime \prime}$ slit rotated to the parallactic angle to minimize the effects of atmospheric dispersion (Filippenko 1982; in addition, LRIS has an atmospheric-dispersion corrector). In our LRIS configuration, coverage in the blue with the $600 / 4000$ grism extends over the wavelength range $3200-5600 \AA$ with a dispersion of $0.63 \AA$ pixel $^{-1}$ and a full width at halfmaximum intensity (FWHM) resolution of $\sim 4 \AA$. We used the $5600 \AA$ dichroic, and our coverage in the red with the $400 / 8500$ grating extends over $5600-10,200 \AA$ with a dispersion of $1.16 \AA$ pixel $^{-1}$ and a resolution of $\mathrm{FWHM} \approx 7$ A. At Gemini, we obtained a spectrum of ASASSN-14gm

4 http://www.astro.washington.edu/users/becker/v2.0/

hotpants.html

5 https://www.aavso.org/apass

6 https://github.com/PaulKuin/uvotpy
(2015 July 27.62) and a spectrum of ASASSN-14dq (2015 Oct. 09) using GMOS. The Gemini spectra were observed with a $1.5^{\prime \prime}$ slit rotated to the parallactic angle using grisms B600 and R400. Details of the spectra and their reduction can be found in Appendix C.

The final light curves of our sample of SNe II are plotted in Figures 1 and 2. The corresponding photometry is provided in the Appendix $\mathrm{C}$.

Table 2 gives more information on our sample including distance modulus, explosion epoch and reddening. To obtain the distance modulus, we use (in order of preference) the Cepheid distance, the expanding photosphere method distance, or the Hubble-flow distance (using a Hubble constant of $73 \mathrm{~km} \mathrm{~s}^{-1} \mathrm{Mpc}^{-1}$ and corrected for Virgo infall). The only exception is ASASSN-14ha, for which the distance modulus ranges from 28.7 to $31.64 \mathrm{mag}$; we decided to use the mean distance modulus of $29.53 \pm 0.5 \mathrm{mag}$ (from NED). This choice makes ASASSN-14ha a low-luminosity SN, similar to SN 2005cs (Pastorello et al. 2005). Giving the extremely low velocities (Arcavi et al. 2014a) and the long plateau, our choice seems appropriate; however, the reader should be aware of the large uncertainty in this value.

The explosion epoch $\left(t_{0}\right)$ is assumed to be the midpoint between the last nondetection and the first detection. The range between these two epochs has been used for the explosion epoch uncertainty. If the last nondetection is not very constraining and the object appears to have been discovered quite young, we estimate an explosion epoch by comparing its spectra to those of similar objects and using the range of epochs of similar spectra for the explosion epoch uncertainty.

Galactic reddening values were obtained from NED as provided by Schlafly \& Finkbeiner (2011). To estimate the host-galaxy reddening we used the equivalent width (EW) of Na I D lines. Gas and dust are often mixed, and some authors have claimed the existence of a statistical correlation between the EW of the Na I D lines and the colour excess (Munari \& Zwitter 1997; Turatto, Benetti \& Cappellaro 2002; Poznanski, Prochaska, \& Bloom 2012). We use spectra collected with the FLOYDS spectrograph, by the Public ESO Spectroscopic Survey of Transient Objects (PESSTO; Smartt et al. 2015) and by the Asiago Transient Classification Programme (Tomasella et al. 2014).

Host-galaxy Na I D lines are not detected in the spectra of SN 2013fs, SN 2013bu, SN 2014cy, LSQ14gv, ASASSN14dq, ASASSN-14gm, ASASSN-14ha, and SN 2015W. In the spectra of SN 2014dw both Galactic and host-galaxy Na I D lines are present with similar EW values, so we set the hostgalaxy reddening equal to the Galactic reddening. PESSTO spectra of SN 2013ai also show host-galaxy Na I D absorption lines that have an EW value twice the Galactic component. Thus, in this case we set the host-galaxy reddening to twice the Galactic reddening ${ }^{7}$.

The FLOYDS spectrum of ASASSN-14kg also shows NaI D lines attributed to the host galaxy with an EW of $1.5 \AA$. Using the equation from Turatto, Benetti \& Cappellaro (2002), this implies a color excess of $E(B-V)_{\text {host }}=0.25 \pm 0.12 \mathrm{mag}$. Using this

7 Here we assume a similar dust-to-gas ratio for NGC 2207 as in the Milky Way. 


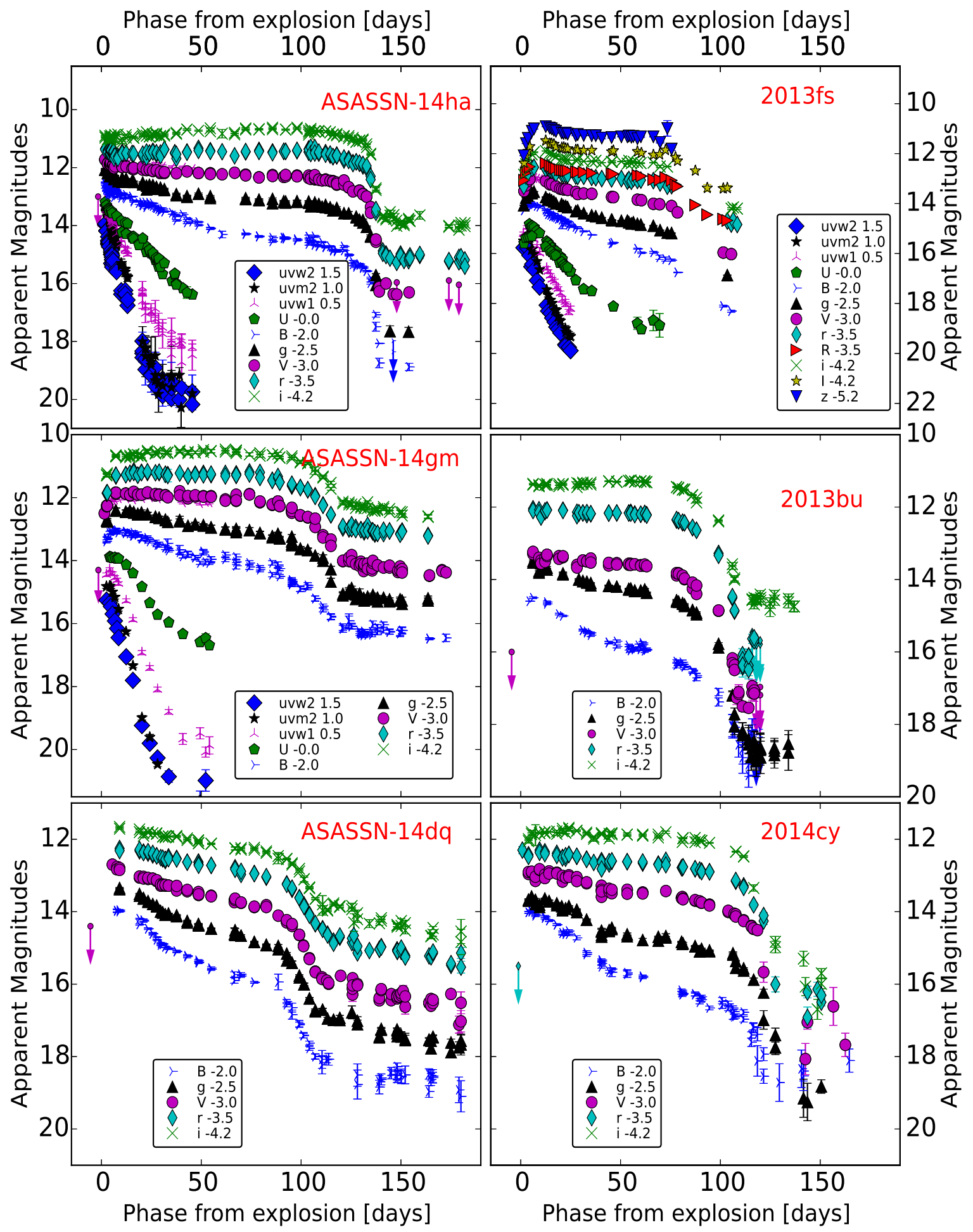

Figure 1. Multiband UV and optical light curves of SNe II observed by LCOGT.

reddening the color of ASASSN-14kg is consistent with that of several other SNe II (corrected for reddening). A larger value would make ASASSN-14kg very blue. Note that in the case of these three SNe we adopt for the host-galaxy reddening a conservative error of $50 \%$ of the inferred value. The only SN for which we do not have optical spectra avail- able to search for the presence of Na I D lines is LSQ13dpa. However, the light curve of this $\mathrm{SN}$ is not red, and it is consistent with the light curves of reddening-corrected SNe II, suggesting that it too is minimally reddened.

The distance modulus, explosion epoch, and reddening 


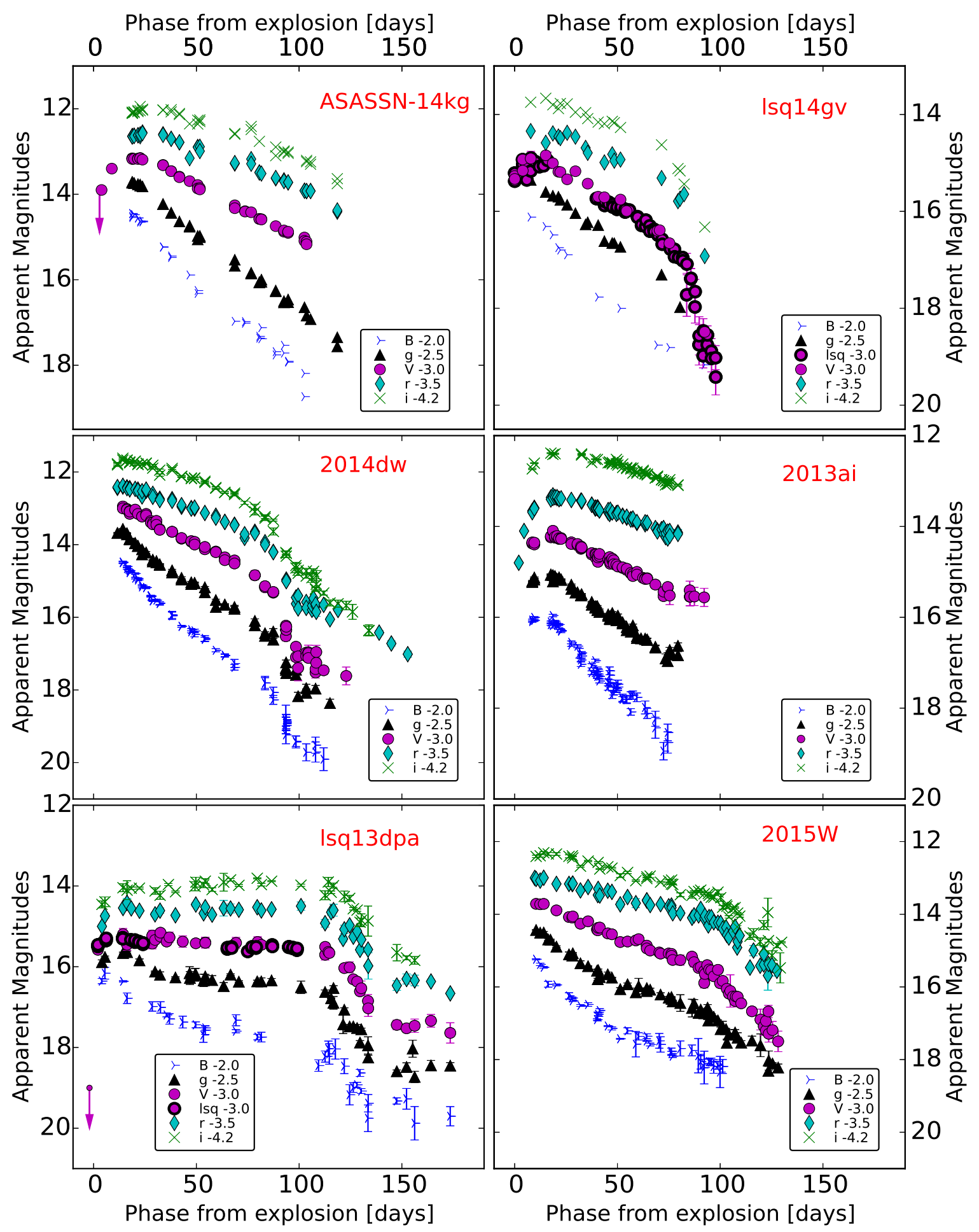

Figure 2. Multiband light curves of SNe II observed by LCOGT. Data from La Silla Quest are marked with a black border. The wide La Silla Quest filter $(r+g)$ is close, but not identical, to Landolt $V$.

of objects in the literature are reported in the online version of Table 2, together with their references.

\section{SNE II: LIGHT-CURVE PARAMETERS}

In this section we introduce several observational parameters to characterize the light curves. The light-curve properties are then compared with those of objects from the literature. 
A graphical visualization of the various light-curve parameters is in Figure 3.

- $s_{50 \mathrm{~V}}$ : decline rate of the $V$-band light curve in mag per 50 days, calculated soon after the light curve reaches the plateau or the linear decay phase. SNe II can take 1-10 days from explosion to reach peak brightness; hence, we calculated the decline rate starting from maximum brightness and extending over 50 days.

- $t_{\mathrm{PT}}$ : this is an indication of the length of the plateau. It is measured from the explosion $\left(t_{0}\right)$ to the midpoint between the end of the plateau phase and start of the radioactive decay-powered $\left({ }^{56} \mathrm{Co} \rightarrow{ }^{56} \mathrm{Fe}\right)$ linear decline phase.

The parameter $t_{\mathrm{PT}}$ is obtained by fitting the $\mathrm{SN}$ light curve in magnitudes around the fall from the plateau with the function

$$
y(t)=\frac{-a 0}{1+e^{\left(t-t_{\mathrm{PT}}\right) / w 0}}+(p 0 \times t)+m 0 .
$$

The fit is computed using a Markov Chain Monte Carlo using the package emcee (Foreman-Mackey et al. 2013). The first term of the equation is a Fermi-Dirac function, which provides a description of the transition between the plateau and radioactive phases ${ }^{8} \cdot t$ is the time from explosion in days. The parameter $a 0$ is the depth of the drop, while $w 0$ measures the slope of the drop (see Fig. 3). The second term of the equation adds a slope to the Fermi-Dirac function to reproduce the light-curve slope before and after the drop. The light curve after the drop is close to the slope of the ${ }^{56}$ Co radioactive decay, while before the drop the light-curve slope may be more heterogeneous. Olivares et al. (2010) and Anderson et al. (2014) used a similar function to estimate $t_{\mathrm{PT}}$ that includes three additional parameters to reproduce the first month of the light curve. However, as we are mainly interested in constraining the time $\left(t_{\mathrm{PT}}\right)$, depth $(a 0)$, and shape $(w 0)$ of the drop, a function with fewer parameters is more appropriate. This allows us to limit our fit to the region close to the drop. The parameter $p 0$, which constrains the slope before and after the drop, is fitted $a$ priori to the light curve after the drop and kept fixed during the fit of the other parameters. For a few objects, the light curve does not extend sufficiently long after the end of the drop to measure $p 0$. For those objects, we fixed $p 0$ to the median value of all the other measurements $(p 0=0.012$ mag per day).

Some SNe II exhibit a change in the slope of the light curve at 10-20 days after maximum. It is only after this time that the photosphere starts to recede deeper than the outer few tenths of a solar mass (Dessart \& Hillier 2010). However, for most SNe II the change in the slope is not clearly visible in a single band, and the light curve can be reproduced by a single slope until the end of hydrogen recombination. To account for this, while studying an expanded sample of $V$ band light curves, Anderson et al. (2014) introduced two additional parameters:

- $s 1$ : the decline rate of the initial, steeper slope of the light curve.

- $s 2$ : the decline rate of the second, shallower slope of the light curve.

8 This function has no direct physical meaning, but it provides a good fit to the transition phase in the light curve.

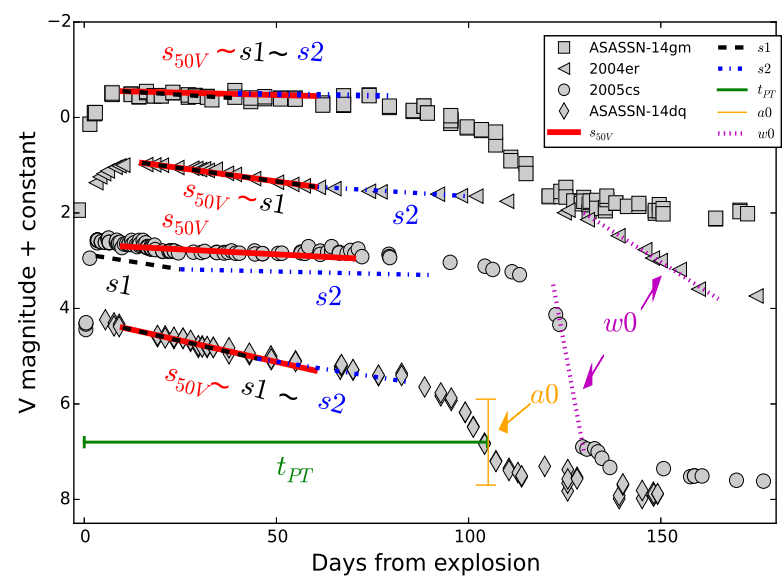

Figure 3. A sample of SN II light curves with the parameters in Section 3 indicated.

To be consistent with our definition of $s_{50 \mathrm{~V}}$, the slopes $s 1$ and $s 2$ are also in units of mag per 50 days. ${ }^{9}$ For objects whose change in slope is not clearly visible, $s 1 \approx s 2 \approx s_{50 \mathrm{~V}}$ (see Fig. 3, ASASSN-14gm and ASASSN-14dq). In several cases the change in slope is visible 30-40 days after explosion (see, e.g., SN 2004er in Fig. 3), and $s 1 \approx s_{50 \mathrm{~V}}$. In some other cases, the change in slope is visible around 20 days after explosion, leading $s_{50 \mathrm{~V}}$ to take on a value between those of $s 1$ and $s 2$ (e.g., SN 2005cs in Fig. 3). While $s 1$ and $s 2$ may highlight differences among SNe II, they are difficult to constrain independently without dense, multiband light-curve coverage. For a single-band analysis, we will mainly use $s_{50 \mathrm{~V}}$, $t_{\mathrm{PT}}, a 0$, and $w 0$. We will later show pseudobolometric light curves that exhibit more pronounced changes in the slope. When measuring the slopes of the pseudobolometric light curves, we will use capitol letters $(S 1$ and $S 2)$ to distinguish these parameters from the single $\mathrm{V}$-band slopes parameters ( $s 1$ and $s 2$ ). Finally, we point out that the end of the plateau is usually defined as the point where the light curve starts to drop from the plateau (OPTd; Anderson et al. 2014). However, the end of the plateau for the majority of the objects occurs $\sim 20$ days prior to $t_{\mathrm{PT}}$, depending on how much the light curve deviates from the linear decay. Given this arbitrary definition, we use $t_{\mathrm{PT}}$ as a measure of the length of the plateau. All of the parameters described above are reported in Appendix D.

In Section 6.3, we will also introduce two additional parameters: the rise time and the magnitude at maximum of the light curve. The rise time of SNe II is relatively fast (1-10 days) compared to the rise times of other types of SNe, and determining it accurately requires good constraints on the explosion epoch and decent photometric coverage at early phases. Some SNe IIP do not actually reach a maximum in their light-curve evolution until the end of the plateau, especially in red passbands. For this reason, we will refer to the light-curve maximum as the last point after which the light curve does not increase by more than 0.1 mag per day (see also Gall et al. 2015 and Rubin et al. 2015).

9 Note that Anderson et al. (2014) instead used mag per 100 days. 
Starting from our sample of $\mathrm{SNe}$ and SNe from the literature, when possible, we measured $s_{50 \mathrm{~V}}$, the rise time, and the magnitude at maximum light in $V, R$ (or $r$ ), and $I$ (or $i$ ). Given the limited number of light curves in $R$ (or $r$ ) and $I$ (or $i$ ) that cover the drop of the linear decay, we measure $t_{\mathrm{PT}}, a 0$, and $w 0$ only in $V$ and with the pseudobolometric light curve. This gives us 45 and 30 objects with information after maximum light and at the moment of the drop in $V$ (see Fig.4) and the pseudobolometric light curve (respectively).

Figure 5 shows several relations among $s_{50 \mathrm{~V}}, a 0, t_{\mathrm{PT}}$, and $w 0$. We used a Pearson test to search for correlations between the measured values of these parameters, and further employed Monte Carlo bootstrapping to investigate the reliability of the test. This is the same approach as previously used by Anderson et al. (2014). No clear clustering for the two distinct SN IIL and SN IIP subtypes is found. A moderate correlation is found between $s_{50 \mathrm{~V}}$ and $t_{\mathrm{PT}}$, with faster-declining SNe II found to have shorter duration plateaus. Anderson et al. (2014) found a similar relation between $s 1$ and OPTd. However, their result was heavily dependent on the extreme case of SN 2006Y. Four of our objects (SN 2013ej, SN 2013by, SN 2013fs, and SN 2014G) show a steep decline and a short $t_{\mathrm{PT}}$, making this correlation more robust. The length of the plateau $\left(t_{\mathrm{PT}}\right)$ is not found to correlate with the slope of the drop $(w 0$; see Fig. $5 \mathrm{~b})$ or the depth of the drop ( $a 0$; see Fig. $5 \mathrm{c})$. The mean length of the plateau is 100 days, while the wide range of the plateau lengths is 60 to 150 days. This is similar to the distribution of OPTd values reported by Anderson et al. (2014) (if we add 20 days to account for the different parameters OPTd and $\left.t_{\mathrm{PT}}\right)$, but it differs from results presented by Poznanski (2013).

An interesting result is that the vast majority of our SNe II (42 out of 45 ) have a drop $(a 0)$ between 1 and 2.6 mag, independent of the slope of the post-maximum decline (see Fig. 5d). The similar drop $(a 0)$ suggests that SNe II with a more luminous plateau should also be more luminous on the radioactive tail. As a consequence, given that the plateau luminosity depends mainly on the kinetic energy of the explosion $\left(L_{\mathrm{SN}} \propto E^{5 / 6}\right.$; Kasen \& Woosley 2009), more-energetic explosions should also produce more nickel. This is in agreement with the relation between expansion velocity and nickel mass reported by several authors (Hamuy 2003; Spiro et al. 2014; Valenti et al. 2015; Pejcha \& Prieto 2015).

Three objects show a significant high $a 0$ : SN 2005cs, SN 2013bu, and ASASSN-14ha. Interestingly, these three objects are low-luminosity SNe II. The relative number of SNe II with a significant drop $(3 / 45)$ is probably a lower limit since we do not have a large number of low-luminosity SNe II in our sample. The peculiar behaviour of these three $\mathrm{SNe}$ is also clear when we correlate with the magnitude at maximum (see Fig. 6); $a 0$ and magnitude at maximum seem to correlate, but the correlation is mainly driven by the large drop of the three faint SNe and the small drop of SN 1979C. In the latter, the drop (if present at all) is around 50 days after maximum. Finally, in the lower panel of Figure 6, we show and confirm the Anderson et al. (2014) result of a clear correlation between $V$-band slope $\left(s_{50 \mathrm{~V}}\right)$ and absolute magnitude.

\section{PSEUDOBOLOMETRIC LIGHT CURVES}

To date, 17 SNe II have multiband photometric coverage extending from near the time of explosion to the radioactivedecay tail. The addition of the current set nearly doubles the published sample. We now proceed to construct pseudobolometric light curves (see Fig.7) (from $U / B$ to $I$ ) for the 30 $\mathrm{SNe}$ II followed beyond the beginning of the radioactivedecay tail.

The transition between the early fast slope $s 1$ and the shallow late slope $s 2$, which is not always visible in a single band, is more evident in the pseudobolometric curves. In Figure 8, we show $s 1$ and $s 2$ measured both for the $V$ band and for the pseudobolometric light curve (the dotted lines connect $V$-band measurements with pseudobolometric measurements). When integrating over the optical range, the light curve becomes steeper at early phases and changes less in the second part of the plateau. At early times, the $\mathrm{SN}$ is expanding and the temperature is dropping, hence, at early time, the bluest bands give large contributions to the light curve. Later, hydrogen recombination starts and the temperature remains close to the recombination temperature $(\sim 6000 \mathrm{~K})$. The pseudobolometric light curve better describes this transition phase.

We have repeated the light-curve analysis using pseudobolometric light curves of $30 \mathrm{SNe}$ II with the function

$$
y(t)=\frac{-A 0}{1+e^{\left(t-T_{\mathrm{PT}}\right) / W 0}}+(P 0 \times t)+M 0 .
$$

The parameters $T_{\mathrm{PT}}, A 0, W 0, P 0$, and $M 0$ are equivalent to the parameters $t_{\mathrm{PT}}, a 0, w 0, p 0$, and $m 0$, but fitting $\log$ (luminosity) as a function of time instead of the $V$-magnitude light curves.

As a first-order approximation to the true bolometric luminosity, we integrated the SN emission in the optical bands $((U) B V R I$ or $(U) B V$ gri, depending on the available bands). All magnitudes were converted to fluxes and integrated using Simpson's Rule (see Valenti et al. 2008, for more details on this method). Given the good light-curve coverage, we measured the parameters $S 1$ and $S 2$, along with $A 0, W 0$, and $T_{\mathrm{PT}}$. Pseudobolometric light curves and the main computed parameters are shown in Figure 10.

No clear correlation is found between $S 1$ and $T_{\mathrm{PT}}$ (see Fig. 9a) owing to the presence of SN 2005cs and SN 2009bw, which have long plateaus and steep $S 1$. A clear correlation between $S 2$ and $T_{\mathrm{PT}}$ is found, confirming that $\mathrm{SNe}$ with a large slope also have a shorter plateau (see Fig. 9b).

\section{EARLY-TIME DATA}

The early-phase emission of SNe II provides clues regarding the nature of the progenitor star at the moment of explosion. For example, the cooling after the shock breakout depends on the ejected mass, energy, and radius of the progenitor (Waxman, Meszaros, \& Campana 2007; Chevalier \& Irwin 2011; Nakar \& Sari 2010). Therefore, a detailed study of SNe II at these phases, particularly with UV and $u B g$ band photometry, may reveal intrinsic differences among the SN II populations. A large fraction of SNe observed with Swift have been published recently (Brown et al. 2009; Pritchard et al 2014; Brown et al. 2014). However, for 


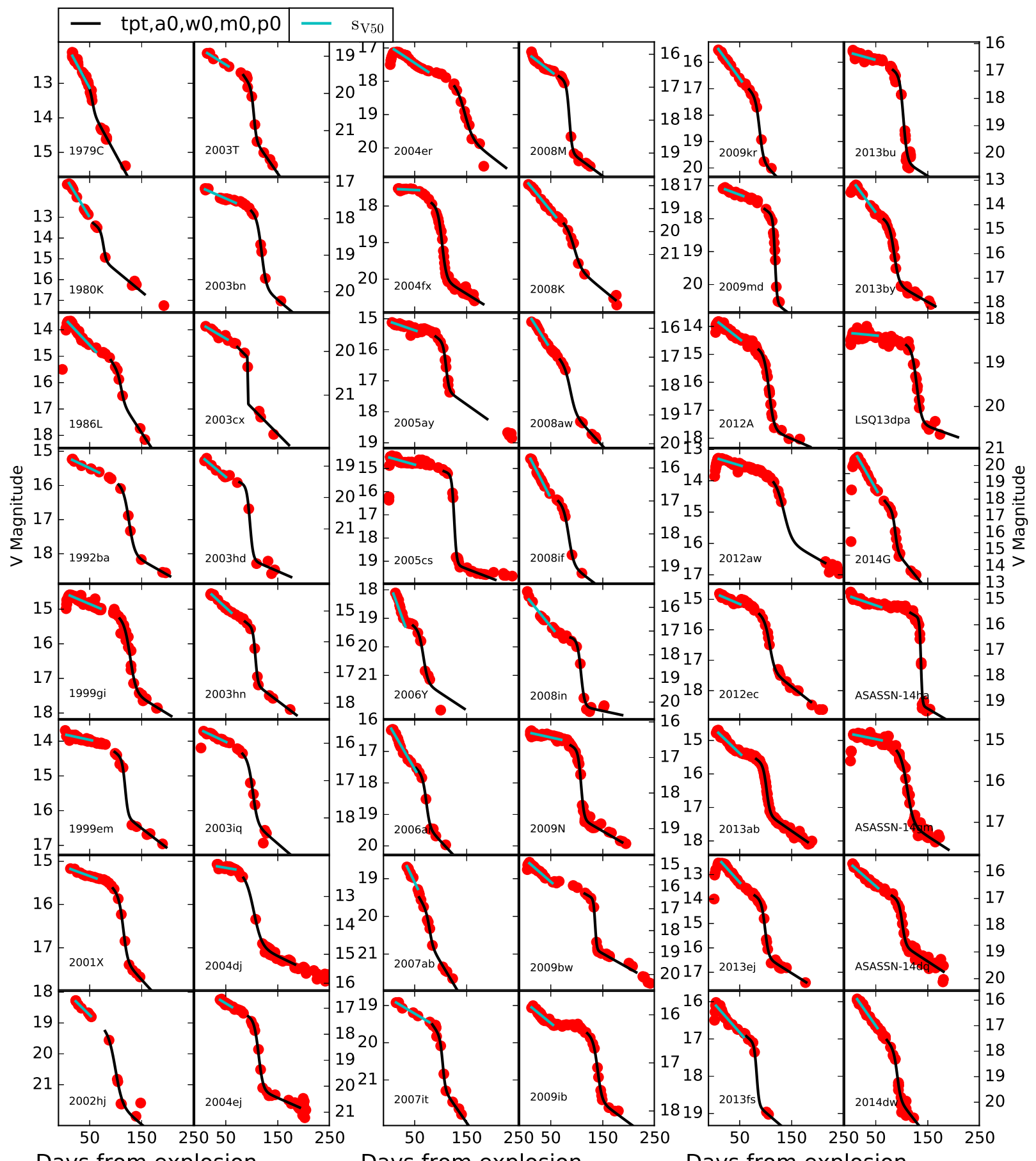

Days from explosion

Days from explosion

Days from explosion

Figure 4. $V$-band light curves and their fits.

SNe IIP/IIL, most of the UV published data are for SNe IIP. In the last two years, several $\mathrm{SNe}$ II have been observed with Swift. Seven (out of 16) SNe in our sample have extended follow-up observations in the UV. Here we present the UV light curves of these SNe, comparing with others available in the literature, with a particular emphasis on determining whether there are intrinsic differences between SNe IIL and IIP. We also show early-time Swift UV spectra of SN 2013ej and compare to those of the nearby, normal Type IIP SN 2012aw (Bayless et al. 2015). 

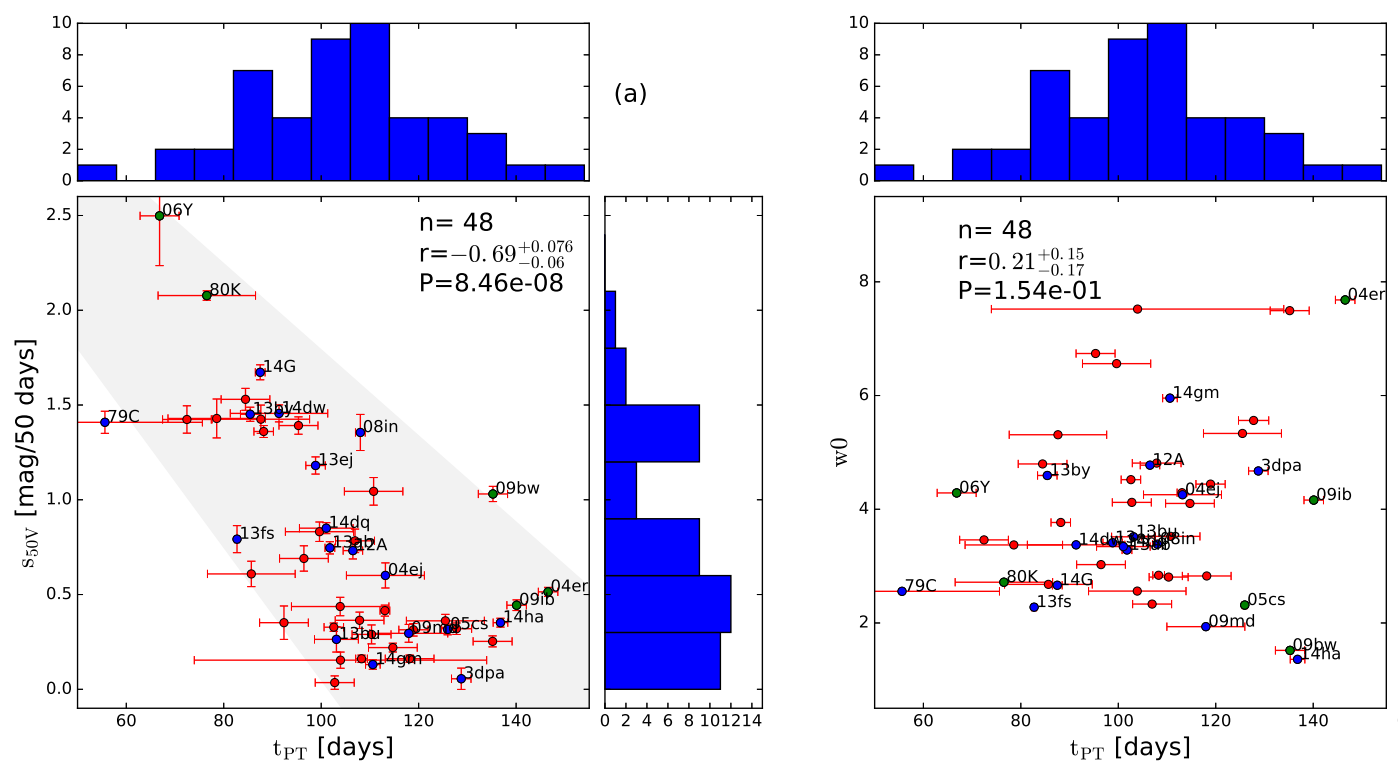

(b)
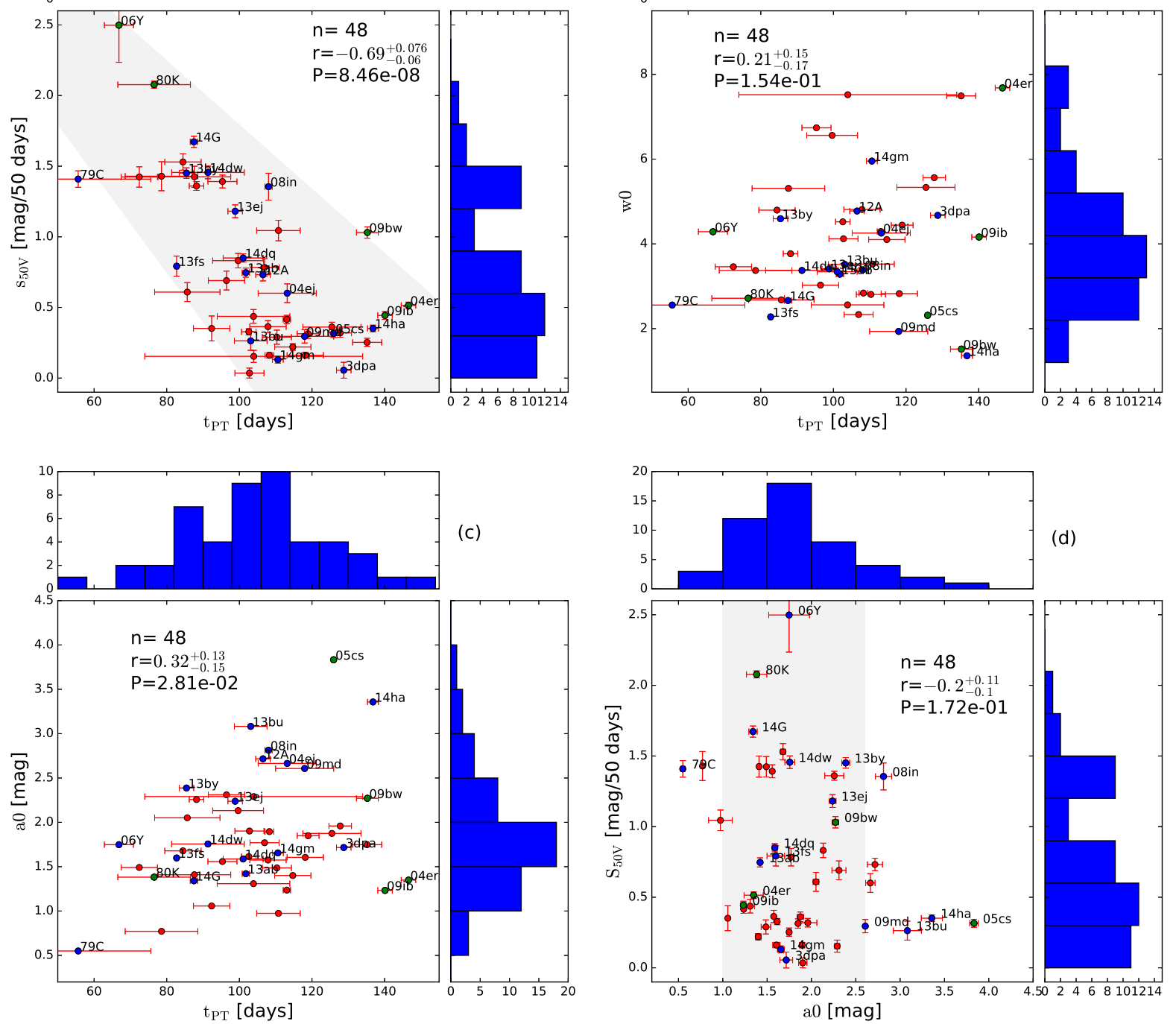

Figure 5. Correlation among $s_{50 V}, t_{\mathrm{PT}}, w 0$, and $a 0$, as obtained from $V$-band light curves. Our sample is marked in blue; some other $\mathrm{SNe}$ are highlighted in green. For each panel $n$ is the number of events, $r$ is the Pearson $s$ correlation coefficient, and $P$ is the probability of detecting a correlation by chance.

\subsection{Colour and Temperature}

The early photospheric temperature evolution of a SN is perhaps the most revealing about the nature of its progenitor (Waxman, Meszaros, \& Campana 2007; Chevalier \& Irwin 2011; Nakar \& Sari 2010). SNe II with RSG progenitors are expected to remain at a relatively higher effective temperature for a longer time than what is expected for SNe II with blue supergiant (BSG) progenitors. A clear example of this is the photospheric temperature of SN 1987A, which was close to $5000 \mathrm{~K}$ (Hamuy et al. 1988) five days after explosion. On the other hand, SN 2013ab and SN 2013ej exhibited temperatures of 10,000 $\mathrm{K}$ at the same epoch, and likely arose from RSG progenitors (Valenti et al. 2014; Bose et al. 2015).
Blinnikov \& Bartunov (1993) suggested that the properties of the SNe IIL 1979C and 1980K may be explained by a large progenitor radius. If SNe IIL arise from progenitors with a large radius and their temperature follows the simple analytic equations of Nakar \& Sari (2010), we should expect to see SNe IIL having higher temperatures than SNe IIP at early phases.

To investigate the photospheric temperature of our sample of $\mathrm{SNe}$, we computed a black-body fit to our multiband photometry ${ }^{10}$. An example of the fit is reported in Figure 11. We will show that the photospheric temperature esti-

10 The magnitudes were converted to flux. Each flux value was assigned to the effective wavelength of each passband. 

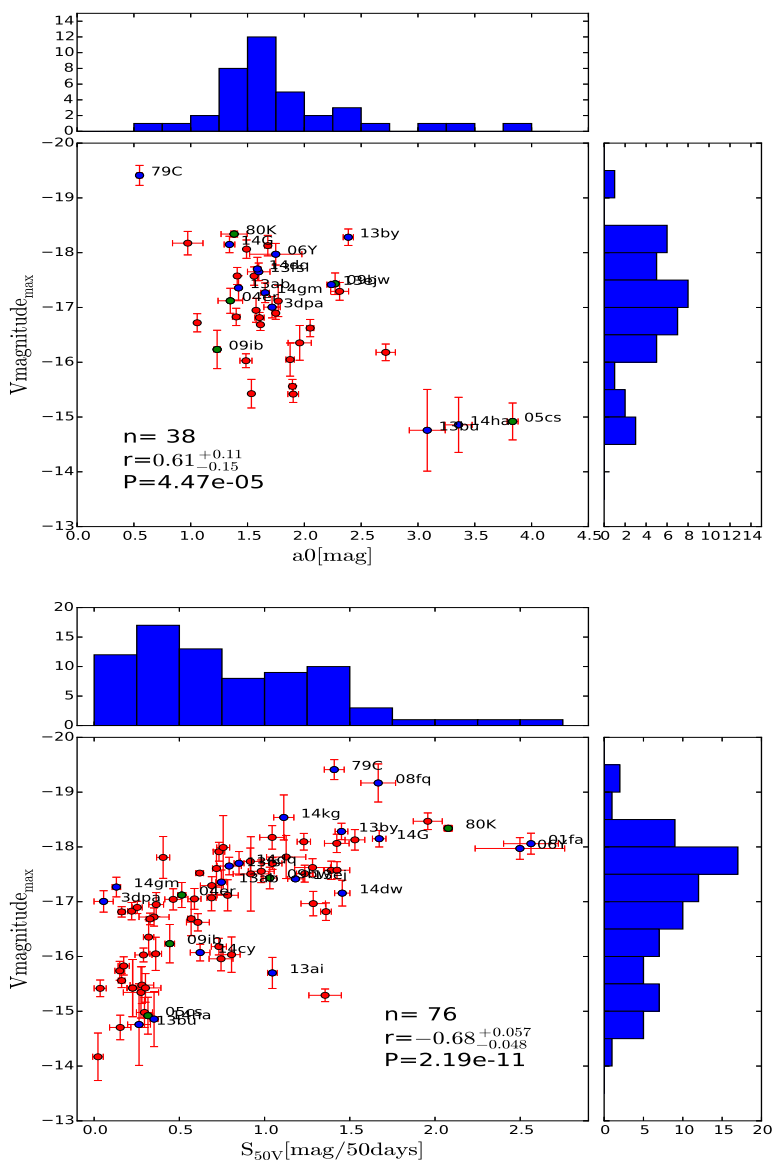

Figure 6. Correlation between absolute magnitude and $a 0$ (top panel) and $s_{50 \mathrm{~V}}$ (lower panel) as obtained from $V$-band light curves.

mated with and without the Swift data can be different. Instead of choosing one or the other, we present the fit both with and without the UV Swift data for the first 30 days.

The temperature evolution of our SN II sample is plotted in Figure 12. Lower and central panels show the temperature evolution (with and without the UV). The objects are colour-coded with respect to the $V$-band decline rate over 50 days $\left(s_{50 V}\right)$. Blue objects are fast-declining SNe IIL. Following the suggestion of Blinnikov \& Bartunov (1993), blue objects should have a higher temperature than red objects. Based on our data, SNe IIL do not always exhibit higher temperatures than SNe IIP.

However, two caveats need to be mentioned. First, the methods presented by Waxman, Meszaros, \& Campana (2007), Chevalier \& Irwin (2011), and Nakar \& Sari (2010) are usually valid only in the first few days after explosion (see also Rubin et al. 2015) when our optical and UV follow-up observations are limited.

A second caveat is that temperature estimates at this early phase are difficult. Valenti et al. (2014) noticed that for SN 2013ej, a cooler temperature was inferred when earlytime UV data are included with the optical. Other SNe II show similar behaviour. The upper panel of Figure 12 shows the temperature evolution of two SNe II with UV data included in the analysis (empty symbols) and without UV

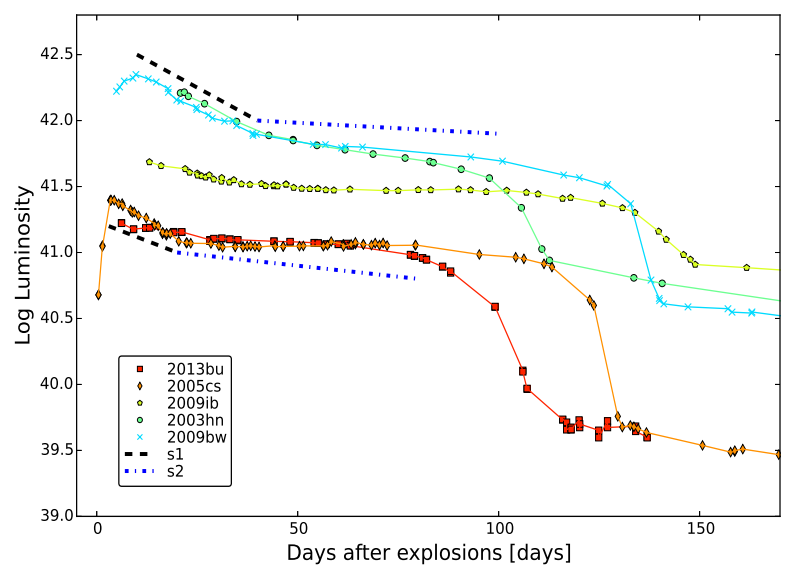

Figure 7. A sample of SN II pseudobolometric light curves.

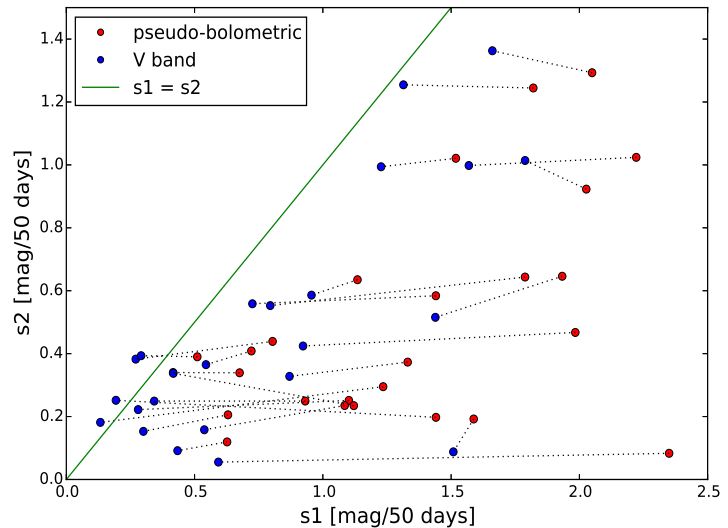

Figure 8. Parameters $s 1$ vs. $s 2$ computed using the $V$ band (blue) and the pseudobolometric light curve (red) for the sample of 22 SNe with multiband coverage. While $s 2$ remains nearly constant, $s 1$ increases if we use the integrated flux.

data (filled symbols). Both objects show a different temperature estimate depending on the spectral energy distribution (SED) extension. In the first few days after explosion, they show a higher temperature if UV data are included. Later, the temperature with only optical bands is higher than the temperature including UV data. It is also important to note that the temperature estimate using only the optical bands is very sensitive at early phases to the $U$ band; at this phase, most of the flux is still emitted in the UV. Ground-based $U$ band data are also notoriously difficult to calibrate (sometimes affected by the atmosphere as well), and may introduce large, poorly understood systematic uncertainties into the temperature estimate. Considering the limited number of SNe IIL available and this $U$-band systematic uncertainty, more high-quality, well-calibrated $U$-band data are needed to properly investigate this issue.

Plotted in Figure 13 is the $U V W 2-V$ broad-band colour evolution for a sample of SNe II. All of these objects have been observed in the UV with Swift beginning soon after their early discovery. A strong change in colour is visible in 

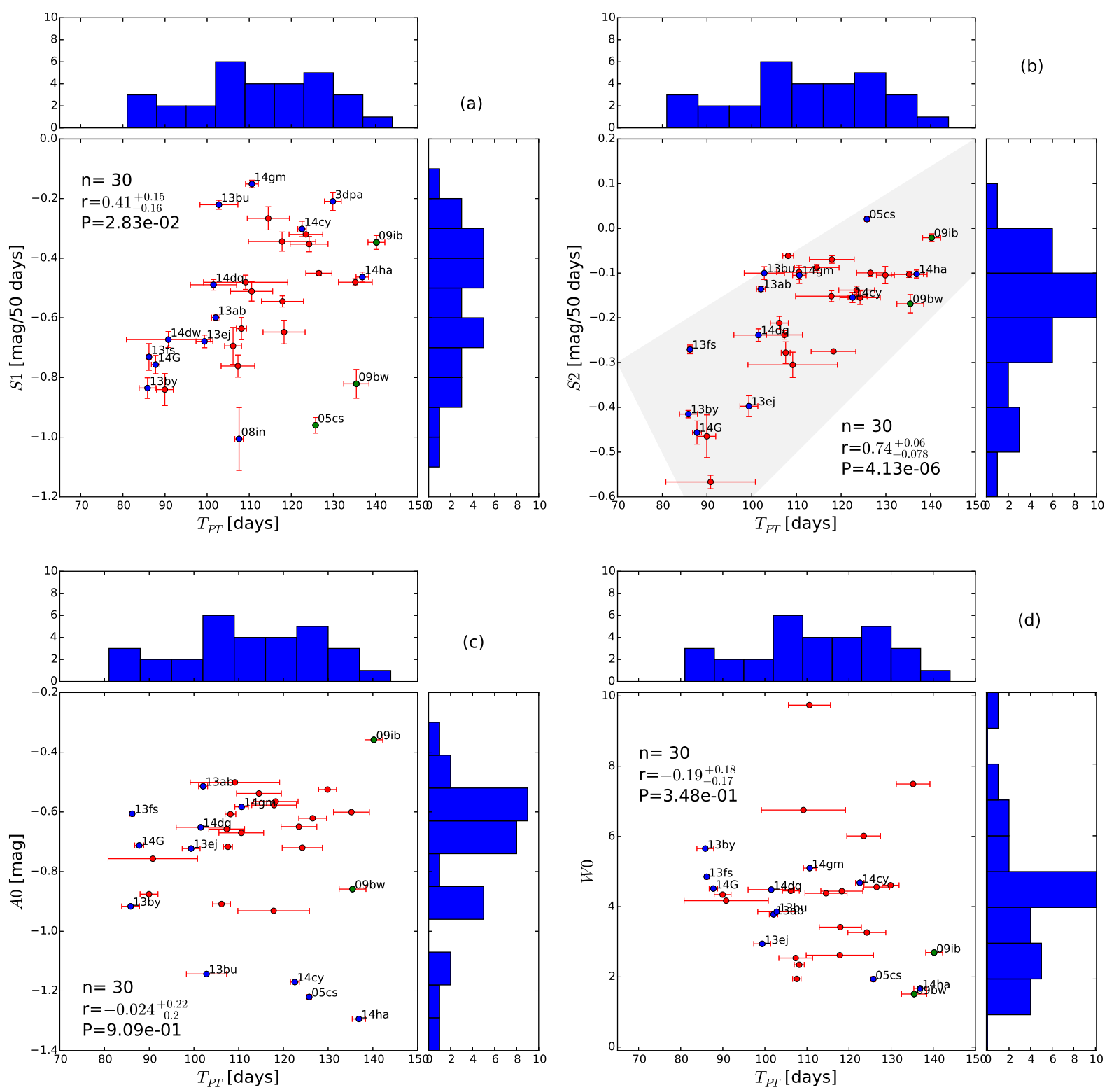

Figure 9. Correlations among $S 1, S 1, T_{\mathrm{PT}}, W 0$, and $A 0$ as obtained from pseudobolometric light curves. Our sample is marked in blue. Some other SNe are highlighted in green. For each panel $n$ is the number of events, $r$ is Pearson's correlation coefficient, and $P$ is the probability of detecting a correlation by chance.

all of the objects regardless of their IIP or IIL classification. This behaviour confirms what we have noticed before: with good photometric precision and knowledge of their explosion epochs, SNe IIP and SNe IIL seem to be indistinguishable based on early-time UV data alone.

\subsection{Ultraviolet Spectra}

In Figure 14 we show Swift UVOT UV spectra of SN 2012aw (top panel) and SN 2013ej (bottom panel). The SN 2012aw spectra have previously been published by Bayless et al. (2013), while the spectra of SN 2013ej were recently presented by Dhungana et al. (2015). As SN 2013ej is a transitional object between a SN IIP and a SN IIL $\left(s_{50 \mathrm{~V}}=1.12\right.$ mag per 50 days), while SN 2012aw is a more typical SN IIP $\left(s_{50 \mathrm{~V}}=0.26 \mathrm{mag}\right.$ per 50 days $)$, direct comparison between these objects may highlight the differences between these SN subtypes.

The UV spectra of these two objects are plotted along with their corresponding visual-wavelength spectra obtained at similar epochs. In both cases, line blanketing is already apparent in the first spectra. In the optical, SNe II can be featureless at early times. However, the UV spectra exhibit several deep and broad absorption features between $2000 \AA$ and $3000 \AA$. In Figure 14, we also show the black-body fit to the optical spectrum (black dashed line) and to the combination of the UV and visual-wavelength spectra (green dotdashed line). Black-body fits yield a higher temperature if 

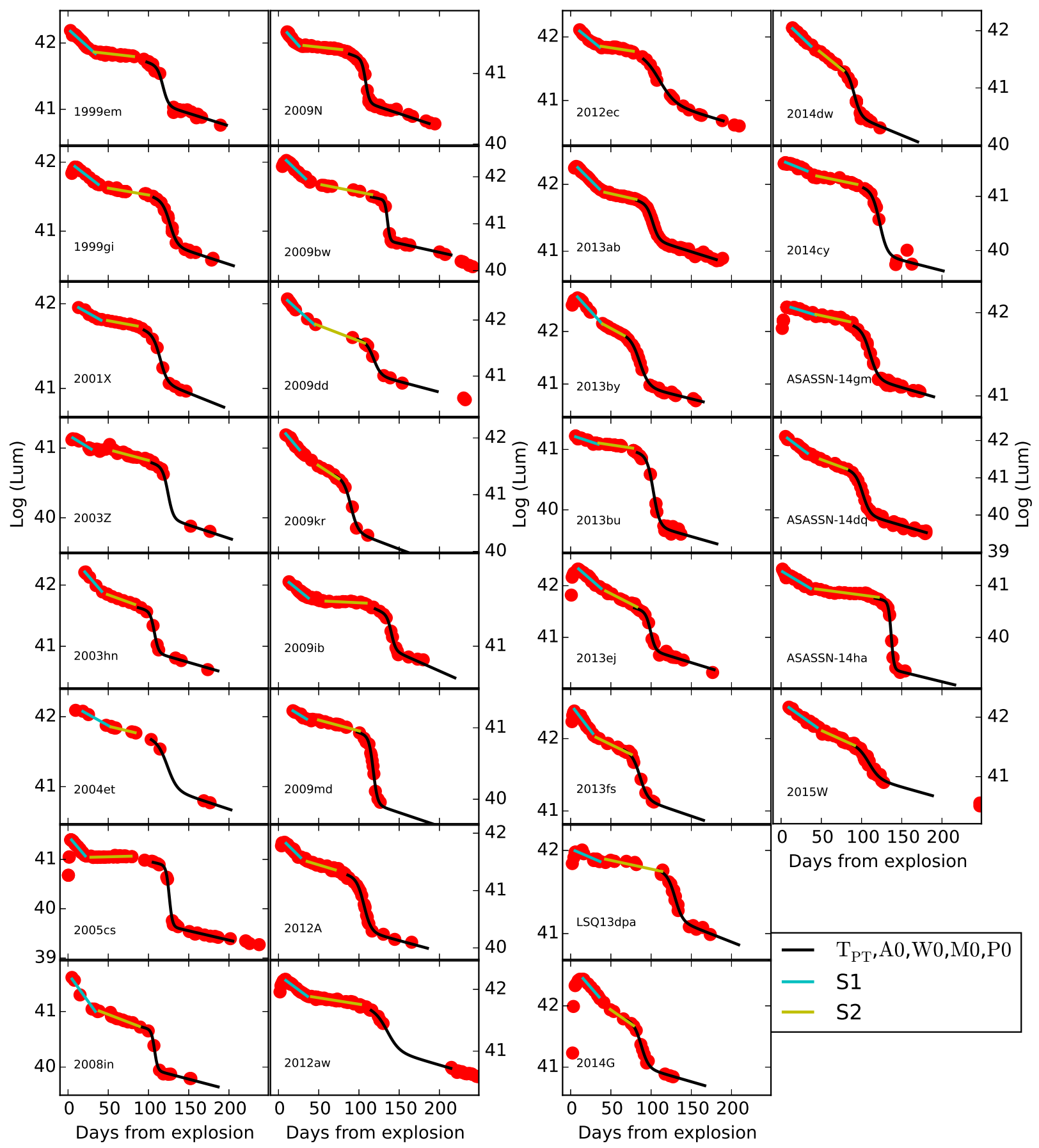

Figure 10. Pseudobolometric light curves and their derived parameters. Data: SN 1999em, Elmhamdi et al. (2003), Leonard et al. (2002a); SN 1999gi, Leonard et al. (2002b), Faran et al. (2014b); SN 2001X, Faran et al. (2014b); SN 2003Z, Spiro et al. (2014); SN 2003hn, Krisciunas et al. (2009); SN 2004et, Maguire et al. (2010); SN 2005cs, Pastorello et al. (2006), Pastorello et al. (2009); SN 2008in, Roy et al. (2011); SN 2009N, Takáts et al. (2014); SN 2009bw, Inserra et al. (2012); SN 2009dd, Inserra et al. (2013); SN 2009kr, Fraser et al. (2009), Elias-Rosa et al. (2010); SN 2009ib, Takats et al. (2015); SN 2009md, Fraser et al. (2011); SN 2012A, Tomasella et al. (2013); SN 2012aw, Dall'Ora et al. (2014); SN 2012ec, Barbarino et al. (2015); SN 2013ab, Bose \& Kumar (2014); SN 2013by, Valenti et al. (2015); SN 2013bu, this paper; SN 2013ej, Yuan et al., in prep.; SN 2013fs, this paper; LSQ13dpa, this paper; SN 2014G, Terreran et al., in prep.; SN 2014dw, this paper; SN 2014cy, this paper; ASASSN-14ha, this paper; ASASSN-14gm, this paper; ASASSN-14dq, this paper; and SN 2015W, this paper. 


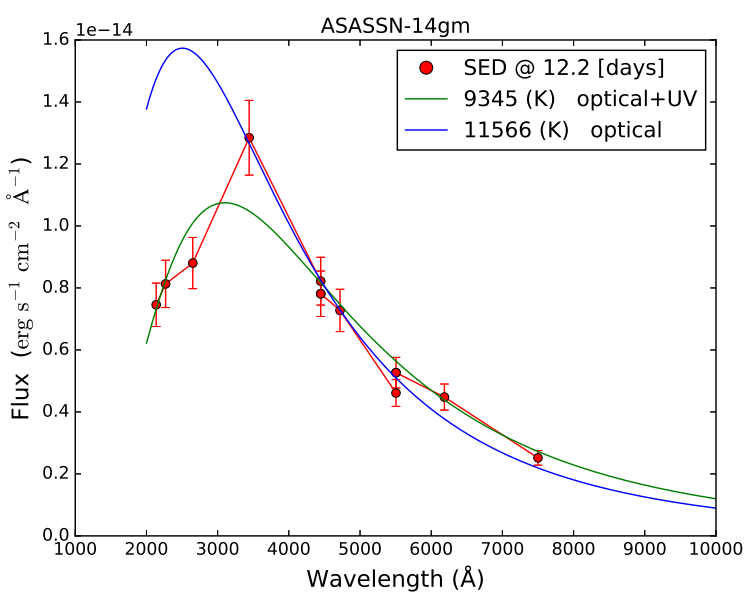

Figure 11. Black-body fit for ASASSN-14gm 12 days after explosion. A different photospheric temperature is obtained with and without the Swift UV filters.

we use only the visual-wavelength spectra, as in Section 6.1. We note that the UV flux should be used with caution to estimate the black-body temperature around one week past explosion, since line blanketing is already prevalent at these epochs.

We made use of SYNOW (Fisher 2000) to identify the lines in the early-time UV spectra (see Fig. 15). Fe II and Ti II are the main ions contributing to the UV features. Co II or Ni II may contribute to the absorption at $\sim 3500 \AA$, although it is difficult to reproduce the depth of the feature without introducing strong Co II and Ni II features blueward of $2200 \AA$. Fe II has also been proposed by Bayless et al. (2013) to explain the line blanketing in the UV spectrum of SN 2012aw, confirming that similar ions characterize the UV spectra of SNe IIP and IIL. We note that the use of SYNOW should be approached with caution owing to limitations in atomic line lists and its one-dimensional non-local-thermodynamicequilibrium nature, making correct line identifications in this wavelength region difficult.

\subsection{Rise Time}

The rise time of SNe II has been a matter of debate. Through the comparison of early-time data of PTF10vdl with those of SN 2006bp (Quimby et al. 2007), Gal-Yam et al. (2011) suggested that SNe II may show a diversity in their rise times. More recently, Valenti et al. (2014) suggested a correlation between rise time and luminosity, with slowly rising SNe II having higher luminosities. Given that SNe IIL are on average more luminous than SNe IIP, and several SNe IIL exhibit a slow rise, there could be a correlation between the rise time and $s_{50 \mathrm{~V}}$ or between the rise time and the peak luminosity.

From a theoretical point of view, such a correlation may be important. Gall et al. (2015), following the equations of Rabinak \& Waxman (2011), pointed out that the rise time of SNe II is proportional to the progenitor radius to the power 0.55 , while it is only marginally dependent on the explosion energy and the ejected mass. They measured the rise time for a sample of $19 \mathrm{SNe}$ II, finding a weak corre-

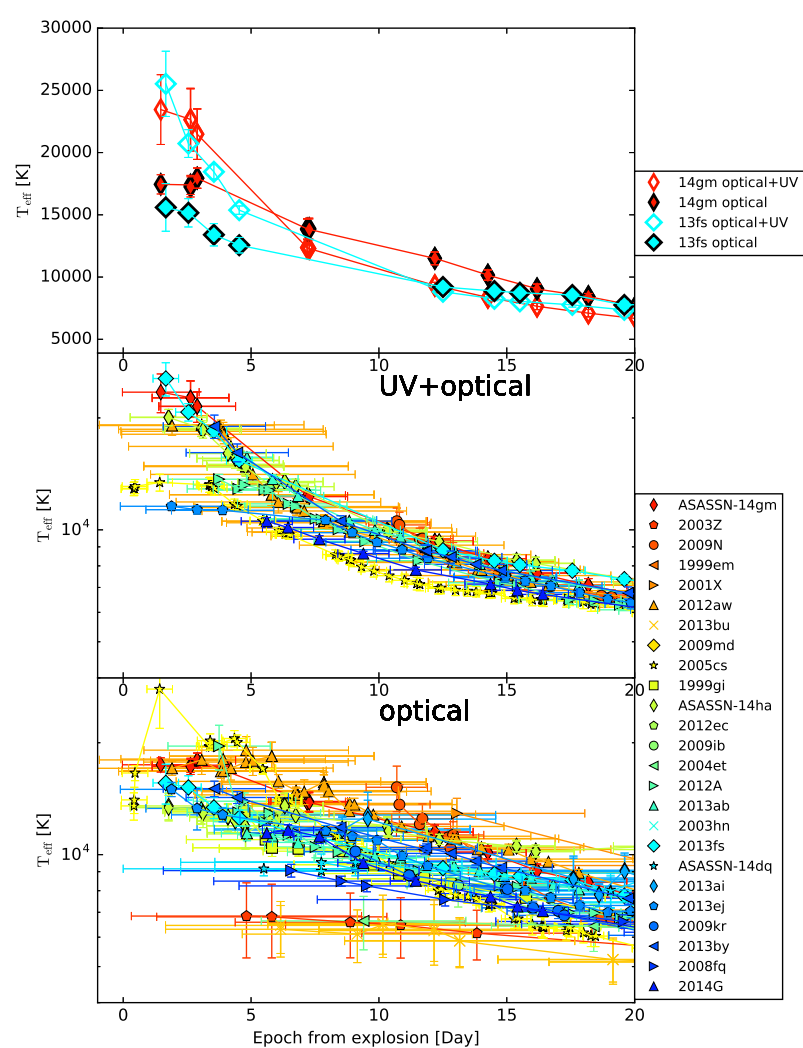

Figure 12. Temperature evolution of a sample of SNe II in the first 20 days. The objects are colour-coded with respect to the $V$-band decline rate over 50 days $\left(s_{50 \mathrm{~V}}\right)$.

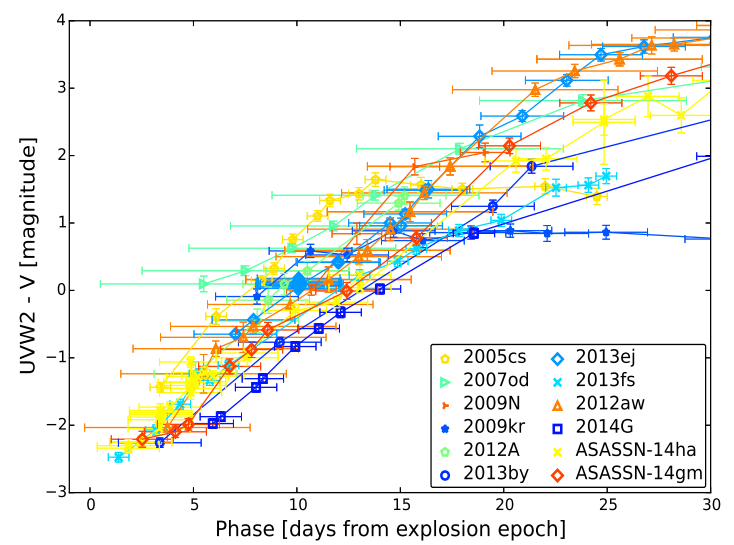

Figure 13. Colour evolution at early phases for a sample of SNe II. Objects from the literature are SN 2012A (Tomasella et al. 2013), SN 2012aw (Bayless et al. 2013; Bose et al. 2013; Dall'Ora et al. 2014), SN 2005cs (Pastorello et al. 2005), SN 2007od (Inserra et al. 2011), SN 2009N (Takáts et al. 2014), and SN 2006bp (Brown et al. 2013). The objects are colour-coded with respect to the $V$-band decline rate over 50 days $\left(s_{50 \mathrm{~V}}\right)$. 

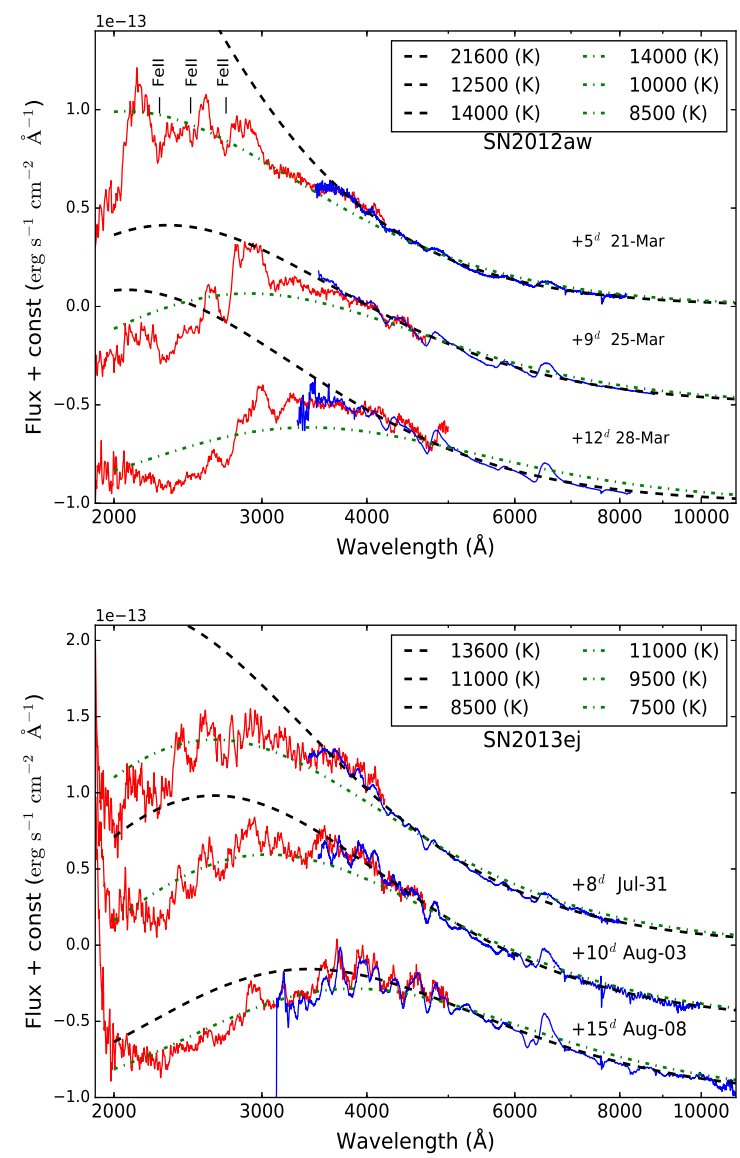

Figure 14. Early-time spectral evolution of the Type IIP SN 2012aw and the (almost) Type IIL SN 2013ej. The red portion of each spectrum corresponds to UV coverage obtained with Swift, while visual-wavelength spectra at similar epochs are plotted in blue. Black-body fits are overplotted as dashed and dotted lines (see text).

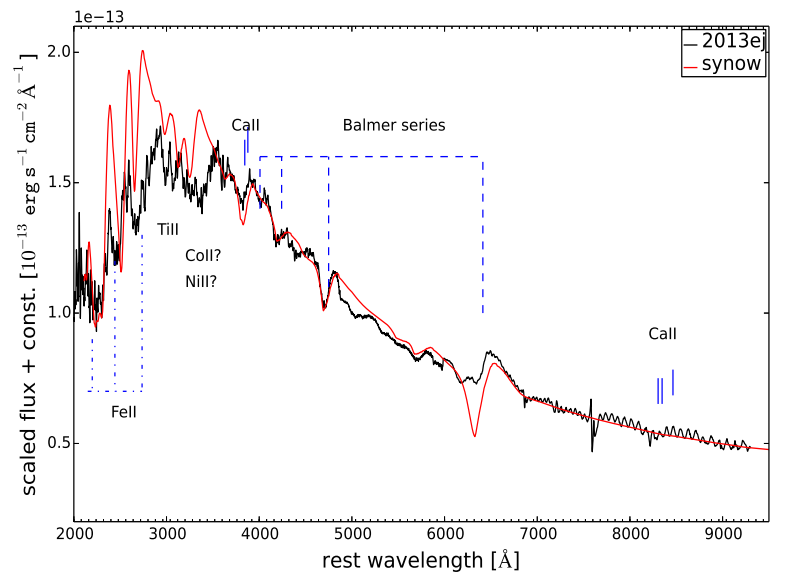

Figure 15. The SYNOW synthetic spectrum is compared with the UV and visual-wavelength spectrum of SN 2013ej. lation between rise time and SN type. Specifically, SNe IIL exhibit a longer rise time than SNe IIP. In a recent paper, Gonzalez-Gaitan et al. (2015) applied a similar analysis to SN II samples from the Sloan Digital Sky Survey II (SDSSII) and the Supernova Legacy Survey (SNLS). Such surveys offer the advantage of having a homogeneous cadence of observations, ideal for doing rise-time studies. Surprisingly, they found the opposite correlation: SNe II that decline slowly after maximum light (SNe IIP) also exhibit a slower rise to maximum, while faster-declining objects (SNe IIL) show a faster rise to maximum light. A third study, using a sample of SNe II discovered by PTF (Rau et al. 2009), also find a weak correlation between rise time and luminosity (Rubin et al. 2015).

A few of our SNe were discovered quite early and may add information to this puzzling question. We applied the technique used by Gall et al. (2015), but excluded SNe with no information within 10 days of explosion (e.g., we excluded SN 1979C for lack of information on the rise time). The rise time is defined as the difference between the explosion epoch and the epoch when the light curve does not change more than 0.01 mag per day. In Figure 16 (top panel), we show the peak luminosity as a function of the computed rise time for a sample of SNe II. They are colour-coded by $s_{50 V}$ : SNe IIL are blue, SNe IIP are red. SNe with no information within 10 days of explosion are reported as upper limits.

We find that the correlation between rise time and luminosity is weak, with a Pearson's correlation coefficient of -0.41 . The weak correlation seems to be caused by the lack of low-luminosity SNe II having a long rise.

As a next step, we investigate the rise time versus the $V$ band decline rate (Fig. 16, bottom panel). Again, the correlation is weak or absent. There are a few objects that clearly have a long rise. Gonzalez-Gaitan et al. (2015) speculated that these slow risers may have a different physical origin. However, we do not see a clear separation from other SNe II.

In summary, using our sample, the correlation found by Gall et al. (2015) is not statistically significant (this is also pointed out by Rubin et al. 2015). The inverse correlation found by Gonzalez-Gaitan et al. (2015) is caused by the fact that their analysis is computed using all filters $(g$, $r, i, z)$ at the same time (private communication). However, Gonzalez-Gaitan et al. (2015) have also shown that rise and decay times are dependent on filters. A reanalysis of the SNLS and SDSS data for each filter is consistent with the absence of a clear correlation (Gonzalez-Gaitan, private communication).

\section{TYPE II SUPERNOVAE AT 50 DAYS}

At 50 days after explosion, the photosphere has receded within the outer $0.5 \mathrm{M}_{\odot}$ of the ejecta (Dessart \& Hillier 2010), and the light curve is mainly powered by hydrogen recombination. This phase is used by several authors to study the SN II diversity. In Figure 17 we investigate the relation between luminosity at 50 days after explosion and length of the plateau, comparing our data with Eq. 18 of Kasen \& Woosley (2009). A weak relation is driven by the fact that faint $\mathrm{SNe}$ tend to have a long plateau, and by SN 1979C which was still very bright 50 days after explosion. The data, all fall below the line representing Eq. 

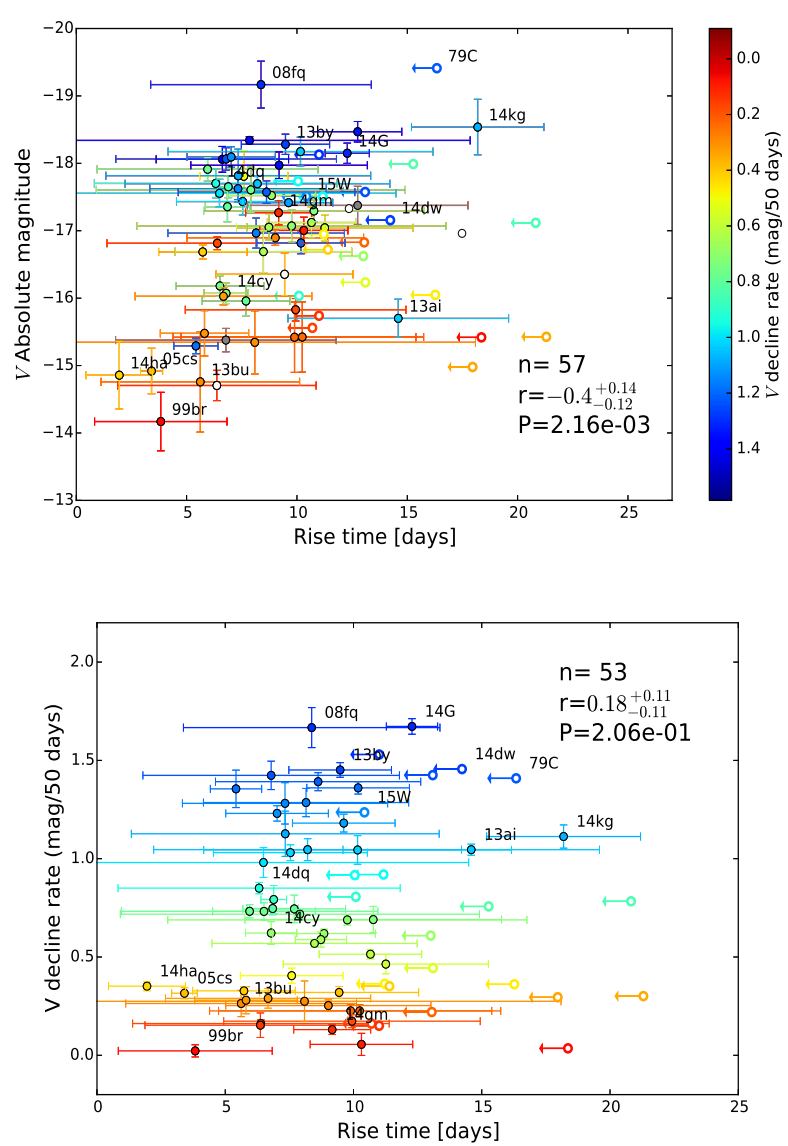

Figure 16. Absolute magnitude as a function of rise time for a sample of SNe II. SNe IIL are blue and SNe IIP are red. For each panel, $n$ is the number of events, $r$ is Pearson's correlation coefficient, and $P$ is the probability of detecting a correlation by chance. The objects are colour-coded with respect to the $V$-band decline rate over 50 days $\left(s_{50 \mathrm{~V}}\right)$.

18 of Kasen \& Woosley (2009). However, the models used in Kasen \& Woosley (2009) are known to overestimate the plateau luminosity (Dessart \& Hillier 2010).

At 50 days, the red part of the spectrum can be approximated by a black body, while iron-group-element line blanketing reduces the flux at the blue end (Kasen \& Woosley 2009). We used bands redder than $V$ (inclusive) to measure an effective temperature at 50 days after explosion. We correlate this temperature with the luminosity at 50 days in Figure 18, color-coding the objects by the $V$-magnitude decline rate. Brighter objects exhibit a higher temperature and IIL-like SNe have systematically lower temperatures. This is probably expected, since IIL-like SNe evolve faster and at 50 days are already close to the end of hydrogen recombination.

\section{NICKEL MASS IN TYPE II SUPERNOVAE}

Recent studies of SNe II have shown that SNe IIL are on average more luminous at peak brightness than SNe IIP (Anderson et al. 2014; Faran et al. 2014a; Sanders et al. $2015)$, though whether they produce more ${ }^{56} \mathrm{Ni}$ has not

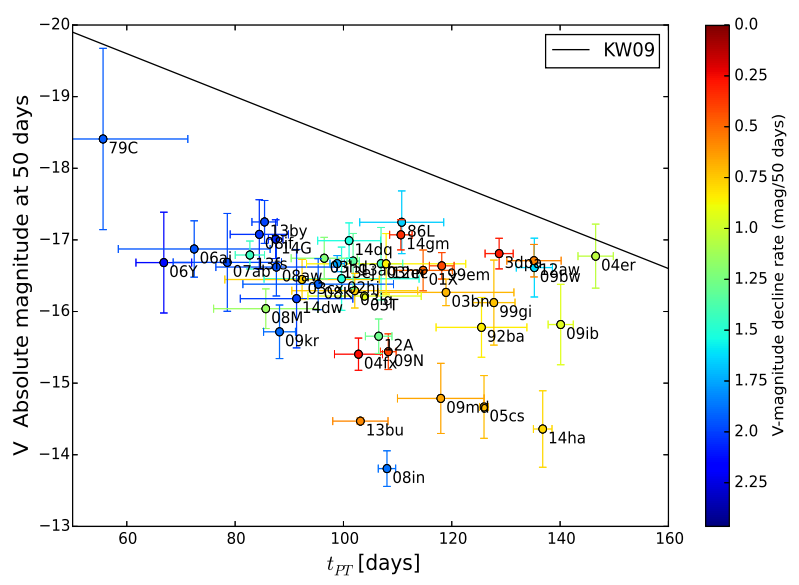

Figure 17. Absolute $V$ magnitude 50 days after explosion vs. plateau length. Objects are colour-coded with the $V$-band decline rate in 50 days. The black line is from Eq. 18 of Kasen \& Woosley (2009).

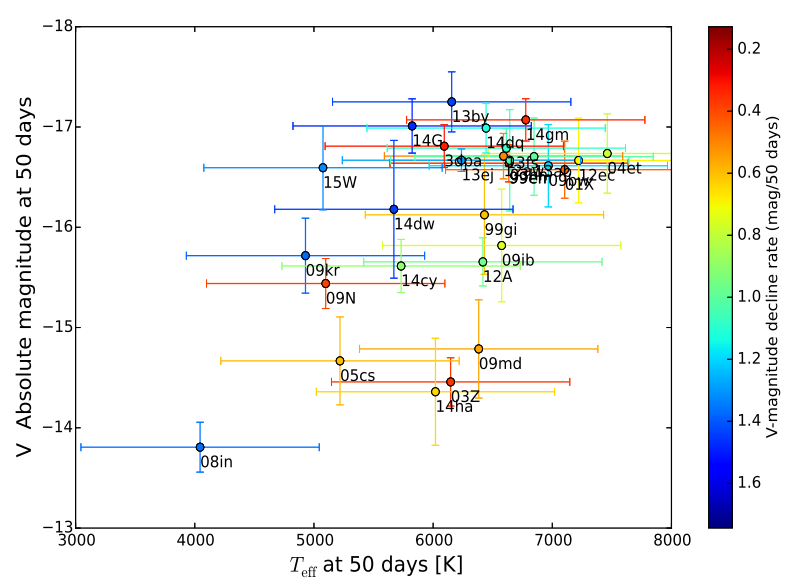

Figure 18. Absolute $V$ magnitude 50 days after explosion vs. $T_{\text {eff }}$. Objects are color-coded with the $V$-band decline rate in 50 days.

been demonstrated. A clear relation between ${ }^{56} \mathrm{Ni}$ content and plateau luminosity has been found (Hamuy 2003; Spiro et al. 2014). Valenti et al. (2015) showed that SNe IIL may follow the same relation, although they increase the scatter. However, Pejcha \& Prieto (2015) have pointed out that luminosity and ${ }^{56} \mathrm{Ni}$ content are strongly correlated, reducing the significance of previous claims.

Theoretically, Popov (1993) and Kasen \& Woosley (2009) have shown that an excess in production of ${ }^{56} \mathrm{Ni}$ may actually produce a longer plateau. Recently, Nakar, Poznanski \& Katz (2015) suggested that the typical ${ }^{56} \mathrm{Ni}$ contribution to the time weighted integrated luminosity during the photospheric phase is $30 \%$. This may be a important factor in the morphology of SN IIP light curves.

Most of our objects were monitored until they reached the ${ }^{56} \mathrm{Co}$ tail, providing an excellent opportunity to better understand how much ${ }^{56} \mathrm{Ni}$ is produced in SNe II. Using SN 1987A as a reference, the ${ }^{56} \mathrm{Ni}$ mass is estimated using the method described by Spiro et al. (2014). To do this, the 


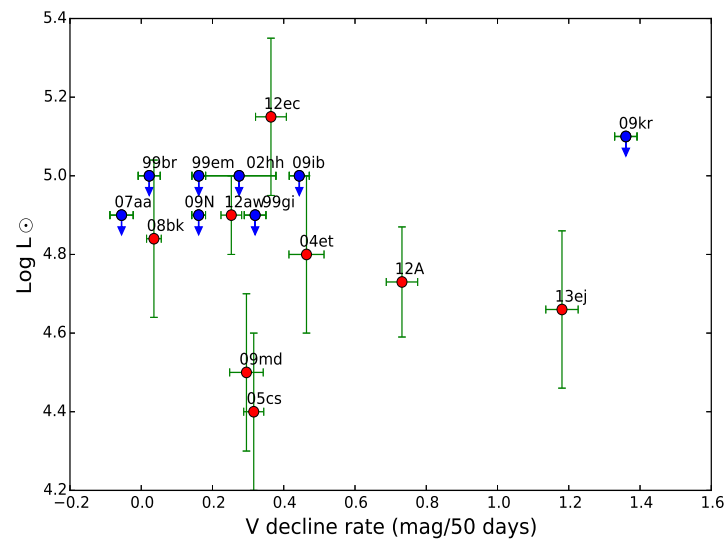

Figure 19. Progenitor luminosity from pre-explosion detection vs. the $V$-band decline rate in 50 days. Progenitor data are from Smartt et al. (2009), Elias-Rosa et al. (2011), Fraser et al. (2011), Fraser et al. (2014), Maund et al. (2015), and Van Dyk et al. (2012).

pseudobolometric light curve of SN 1987A is compared with those of our sample when the objects are on the radioactive tail $\left(t>t_{P T}\right)$. Note that the pseudobolometric light curve of SN 1987A used for comparison was constructed with the same limits of integration ${ }^{11}$ and over similar epochs as was done for the objects in our sample. The comparison of SN 1987A to any given object yields a ${ }^{56} \mathrm{Ni}$ mass estimate following the relation

$$
M\left({ }^{56} \mathrm{Ni}\right)=0.075 \mathrm{M}_{\odot} \times \frac{L_{\mathrm{SN}}(t)}{L_{87 \mathrm{~A}}(t)} .
$$

We next investigate four questions: (1) Do more-massive progenitors produce SNe IIL? (2) Is the amount of ${ }^{56} \mathrm{Ni}$ correlated with the luminosity decay rate? (3) Is the ${ }^{56} \mathrm{Ni}$ content correlated with the length of the plateau, as claimed by Popov (1993) and Kasen \& Woosley (2009)? (4) Do moreluminous SNe II produce more ${ }^{56} \mathrm{Ni}$ ?

The current dataset suggests that the answer to the first question is negative. However, the number of SNe II with a progenitor detection in archival images and a measurement of the decay rate on the plateau is still limited. With the data available today, we do not see any evidence that SNe IIL are related to a more-massive progenitor (see Fig. 19). We will come back to this issue in the next section.

For the second question, we have a statistically significant sample of SNe II (see Fig. 20) and the lack of a correlation is robust.

There is no correlation between ${ }^{56} \mathrm{Ni}$ mass and the length of the plateau (see Fig. 21). A possible explanation comes from the models of Popov (1993) and Kasen \& Woosley (2009), which show that ${ }^{56} \mathrm{Ni}$ begins to contribute to the plateau length only when large amounts $\left(\geqslant 0.06 \mathrm{M}_{\odot}\right)$ of ${ }^{56} \mathrm{Ni}$ are produced. For $\mathrm{SNe}$ that produce less ${ }^{56} \mathrm{Ni}$, the effect is usually negligible. The number of SNe II that produce more than $0.06 \mathrm{M}_{\odot}$ of ${ }^{56} \mathrm{Ni}$ is very small. We

$11(U) B V R I$ or $(U) B V g r i$, depending on the available bands

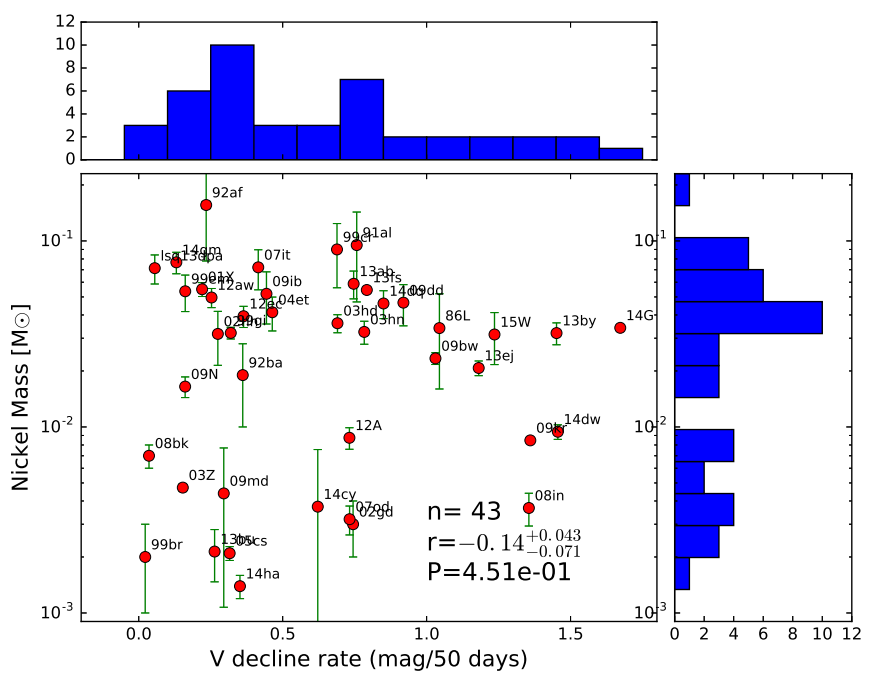

Figure 20. ${ }^{56} \mathrm{Ni}$ mass for a sample of SNe II vs. the $V$-band decline rate in 50 days for a sample of SNe II. The data for the sample include SNe II from Hamuy (2003) and Spiro et al. (2014). For each panel, $n$ is the number of events, $r$ is Pearson's correlation coefficient, and $P$ is the probability of detecting a correlation by chance.

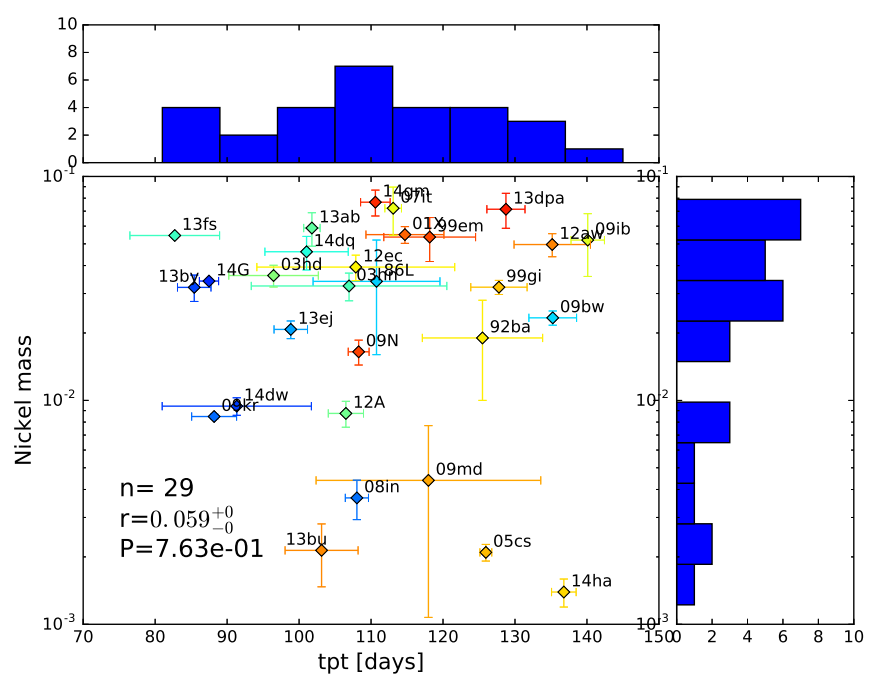

Figure 21. ${ }^{56} \mathrm{Ni}$ mass as a function of time of the transition between the plateau and radioactive tail; $n$ is the number of events, $r$ is Pearson's correlation coefficient, and $P$ is the probability of detecting a correlation by chance. The objects are colour-coded with respect to the $V$-band decline rate over 50 days $\left(s_{50 \mathrm{~V}}\right)$.

note that the length of the plateau also depends on the energy of the explosion and the initial radius (see Eq. 11 of Kasen \& Woosley 2009). This may partially contribute to the lack of a correlation between ${ }^{56} \mathrm{Ni}$ mass and the length of the plateau. Fig. 20 and Fig 21 show however a lack of SNe that produce $\left.10^{-2} \mathrm{M}_{\odot}\right)$ of ${ }^{56} \mathrm{Ni}$.

Fig. 22, confirm the finding (Hamuy 2003; Spiro et al. 2014; Pejcha \& Prieto 2015) that brighter SNe produce 


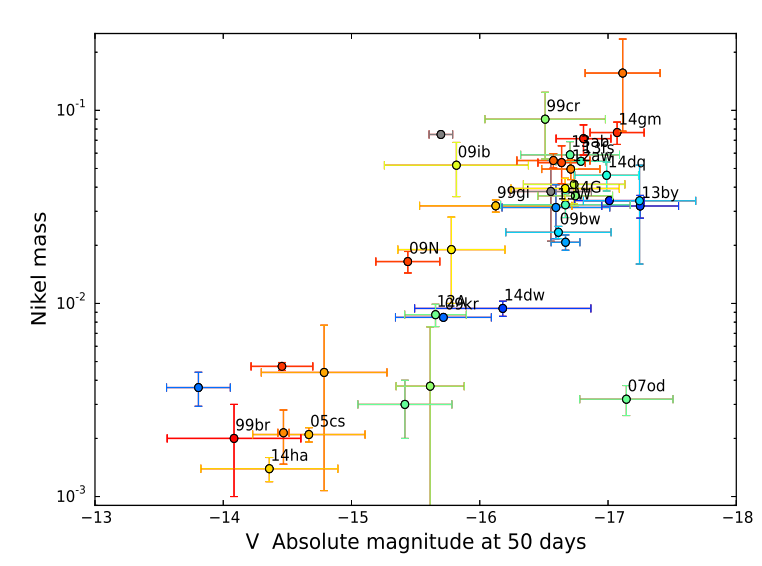

Figure 22. ${ }^{56} \mathrm{Ni}$ mass as a function of the absolute $V$-band magnitude at 50 days after maximum The objects are colour-coded with respect to the $V$-band decline rate over 50 days $\left(s_{50 \mathrm{~V}}\right)$.

larger ${ }^{56} \mathrm{Ni}$ masses. However the scatter in the relation is also suggesting that a simplistic two-dimensional parameter space plateau luminosity - ${ }^{56} \mathrm{Ni}$ mass is probably an over simplification of the problem.

\section{NEBULAR SPECTRA}

An alternative way to constrain the nature of $\mathrm{SNe}$ II is trough the study of their late-phase ( $>200$ days after explosion) nebular spectra. At this phase, SN II ejecta have sufficiently expanded that they become transparent to optical light (e.g., Fransson \& Chevalier 1989; Taubenberger et al. 2009; Maguire et al. 2012).

The nucleosynthesis during the last phases of massivestar evolution produces a carbon-oxygen core with an elemental composition that is strongly dependent on mass. For example, the amount of oxygen produced in stellar evolutionary models varies from 0.2 to $5 \mathrm{M}_{\odot}$ for progenitors between 10 and $30 \mathrm{M}_{\odot}$ (e.g., Woosley \& Heger 2007). These differences should be visible (see Jerkstrand et al. 2014 for a discussion), and spectral synthesis modeling has been widely used to constrain the abundance of different elements in the ejecta (Mazzali et al. 2007, 2010; Dessart \& Hillier 2010; Dessart et al. 2013; Jerkstrand et al. 2012, 2015a,b) and to elucidate the nature of the progenitor.

Jerkstrand, Fransson \& Kozma Jerkstrand et al. (2012) developed quantitative method of taking the ejecta stratification structure of a stellar model at the moment of explosion (e.g., the models of Woosley \& Heger 2007), carrying out a detailed calculation of the energy deposition of gamma-rays/positrons by radioactive isotopes, and combining this with radiativetransfer calculations to produce synthetic nebular spectra. The computed synthetic spectra can then be compared with the observed spectra at different phases.

By analyzing the synthetic spectra from different progenitors, Jerkstrand et al. (2014) found that the intensities of specific lines are correlated with the progenitor mass. In particular, [O I] $\lambda \lambda 6300,6364$ is a fare indicator of the progenitor mass. By measuring the [O I] intensity,

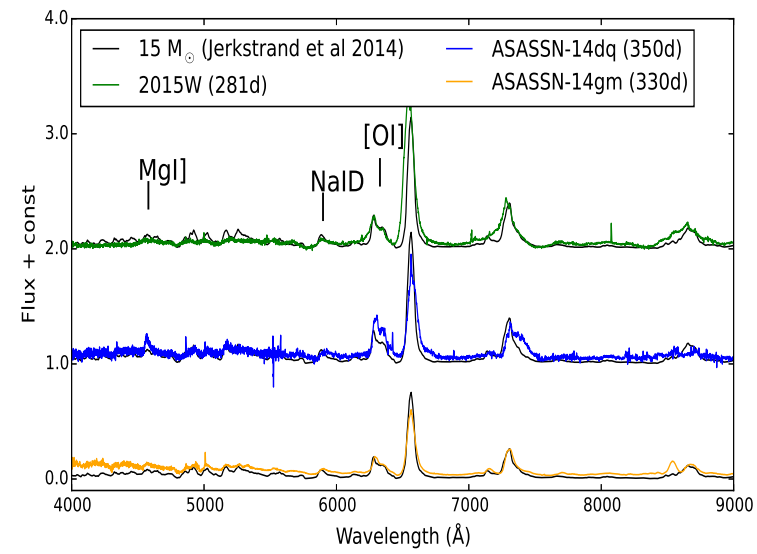

Figure 23. Nebular spectra of three SNe II from our sample. The intensity of [O I] in all three $\mathrm{SNe}$ is consistent with the Jerkstrand et al. 2015b model of $15 \mathrm{M}_{\odot}$.

Jerkstrand et al. (2015b) found that most SNe II are consistent with progenitor stars with mass $\leqslant 17 \mathrm{M}_{\odot}$. This is consistent with progenitor studies using archival prediscovery images: SNe II likely come from progenitors in the range 8-16 $\mathrm{M}_{\odot}$ (Smartt et al. 2009; Smartt 2015). As noted previously, the lack of SN II progenitor detections with large masses is called the RSG problem. Nebular-phase spectroscopy allows us to investigate whether SNe IIL come from more-massive progenitors than SNe IIP. We stress, however, that this type of nebular-spectrum analysis relies on the assumption that SNe II have roughly similar values for certain parameters (e.g., explosion energy, mixing, mass loss).

We collected six spectra for three of the objects in our sample. These spectra are shown in Figure 23, and detailed information is reported in Appendix C.

We show the Jerkstrand et al. (2015b) diagram in Figure 24 with three more objects added. ASASSN-14dq and SN 2015W are two fast-declining SNe II and the oxygen profiles in their spectra are consistent with progenitors of $\sim 15 \mathrm{M}_{\odot}$, similar to other SNe IIP. Figures 23 and 24 show that these two SNe II (which would be reasonably classified as SNe IIL) do not show significant differences compared to the $15 \mathrm{M}_{\odot}$ models. In particular, the oxygen line strengths are clearly not greater than either the model spectra or the bulk of the SNe IIP observed to date (see also Figure D4). While a larger sample is required before making definitive conclusions, the simple interpretation of our data is that SNe IIL come from progenitors having core helium masses that are no more massive than those of SNe IIP. Whether massive RSGs can explode as SNe IIL remains an open question, but there is no evidence from our data that they come from stars more massive than $\sim 17 \mathrm{M}_{\odot}$, and so we cannot solve the RSG problem elucidated by Smartt et al. (2009).

\section{DISCUSSION AND CONCLUSIONS}

We have presented extensive multiband photometry for a sample of 16 SNe II. The light curves follow the flux evolution of our sample out to the radioactive ${ }^{56} \mathrm{Ni}$ decay tail. Main parameters (e.g., the rise time to peak brightness, the 


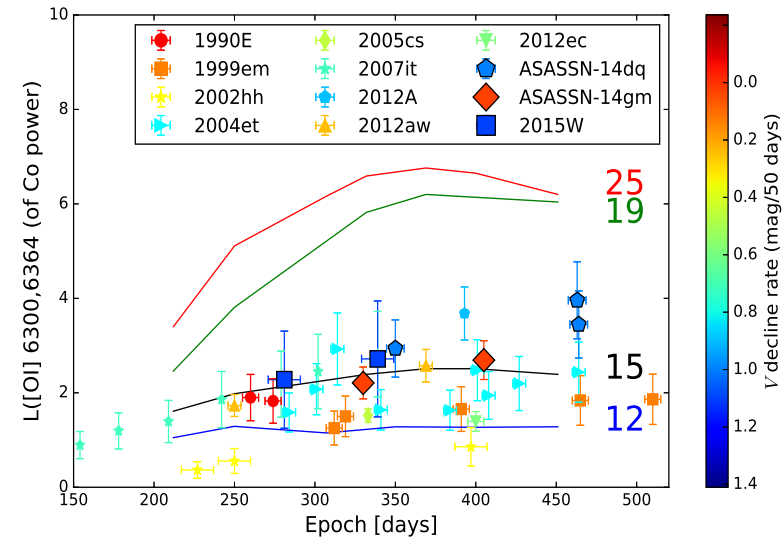

Figure 24. The [O I] $\lambda \lambda 6300,6364$ luminosity (normalized to the ${ }^{56}$ Co decay power) for the Jerkstrand et al. (2015b) sample of $\mathrm{SNe}$ and three objects from our sample. Objects are colour-coded with the $V$-band decline rate $s_{50} \mathrm{~V}$.

plateau length, and the decay slope) are estimated from both the $V$-band light curves and pseudobolometric light curves. These results are placed into context with an expanded sample drawn from the literature.

Our main findings can be summarized as follows.

- SNe II exhibit some differences in their light-curve slopes, plateau lengths, and absolute magnitudes. However, we do not find a clear separation between these subtypes that would justify the historical classification into distinct Type IIP and Type IIL subclasses.

- SNe IIP and SNe IIL do not exhibit significant temperature differences at early phases. This suggests that SN IIL progenitors do not have systematically larger radii, as confirmed by the early-time colour evolution. We also find that temperature evolution studies at early phases have several potential pitfalls. The approximation by a black-body function is problematic even at a few days after explosion, as strong line blanketing in the UV strongly effects the SED.

- We confirm that there is a correlation between the plateau length and the slope of the light curve after maximum light. The correlation is significant with the $s_{50 \mathrm{~V}}$ parameter (in $V$ ) and with the $S 2$ parameter (using the pseudobolometric light curve).

- SNe II have a drop from the plateau to the radioactive decay of 1.0-2.6 mag, which appears to be independent of the length of the plateau confirming that brighter plateau have more ${ }^{56} \mathrm{Ni}$. The three SNe with a large drop (3-4 mag) are low-luminosity SNe.

- We measure the rise time of SNe II using the approach adopted by Gall et al. (2015). We find a weak correlation between rise time and luminosity, while no correlation is found between rise time and light-curve decline.

- We investigate the possibility that SNe IIL come from more-massive progenitors. Despite the limited amount of data on the luminosity of the progenitors and the number of nebular spectra of SNe IIL, so far there is no conclusive evidence that the mass of the progenitors of SNe II correlates with the slope of the decline rate after maximum light.

- We find that the plateau luminosity at 50 days after maximum correlates with the plateau length and with the effective temperature.

- Despite the fact that SNe IIL are on average more luminous than SNe IIP (Patat et al. 1994; Li et al. 2011), there is no clear evidence that the decline rates of $\mathrm{SNe}$ II correlate with the amount of ${ }^{56} \mathrm{Ni}$ observed.

Finally, our analysis (in $V$ and pseudobolometric light curve) is consistent with the result of Anderson et al. (2014) and Sanders et al. (2015): SNe IIP and SNe IIL cannot be comfortably separated in two different classes. Similar investigations in $R$ or $I$ are, however, still limited by the small number of SNe published, but so far, are in agreement with the result in $V$ band (see Fig. D1 and Galbany et al. 2015).

We find no clear evidence that SNe II with a fast lightcurve decline (SNe IIL) originate from a more massive progenitor. While SNe IIP and SNe IIL cannot be comfortably separated in two different classes, faint SNe II behave often differently from the rest of SNe II (eg. plateau length and ${ }^{56} \mathrm{Ni}$ synthesized). However the lack of $\mathrm{SNe}$ between faint and normal SNe II may still due to the small sample of SNe.

The construction of a larger sample of SN II progenitors and a more thorough statistical study of their late-time oxygen lines (used as a progenitor-mass tracer) is required to increase our understanding of SNe II progenitors.

\section{ACKNOWLEDGEMENTS}

The authors acknowledge the ASASSN, La Silla Quest, and LOSS surveys for discovering new SNe that made this study possible. This material is based upon work supported by the National Science Foundation (NSF) under Grant No. 1313484. M.D.S. gratefully acknowledges generous support provided by the Danish Agency for Science and Technology and Innovation realized through a Sapere Aude Level 2 grant. M.F. is supported by the European Union FP7 programme through ERC grant number 320360. S.J.S. acknowledges funding from the European Research Council under the European Union's Seventh Framework Programme (FP7/2007-2013)/ERC Grant agreement No. [291222] and STFC grants ST/I001123/1 and ST/L000709/1. A.V.F.'s group at UC Berkeley is grateful for financial assistance from NSF grant AST-1211916, the TABASGO Foundation, Gary and Cynthia Bengier, and the Christopher R. Redlich Fund. This work was supported by the NSF under grants PHY-1125915 and AST-1109174. M.S. acknowledges support from EU/FP7-ERC grant no [615929]. This paper is based on observations made with the Swift, LCOGT, Gemini, and Keck Observatories; we thank their respective staffs for excellent assistance. The W. M. Keck Observatory is operated as a scientific partnership among the California Institute of Technology, the University of California, and NASA; the observatory was made possible by the generous financial support of the W. M. Keck Foundation. Based on observations collected at the European Organisation for Astronomical Research in the Southern Hemisphere, Chile as part of PESSTO, (the Public ESO Spectroscopic Survey for Transient Objects Survey) ESO program ID 188.D-3003. 


\section{REFERENCES}

Anderson J. P. et al., 2014, ApJ, 786, 67

Andrews J. E. et al., 2011, ApJ, 731, 47

Arcavi I. et al., 2012, ApJ, 756, L30

Arcavi I., Hosseinzadeh G., Valenti S., Howell D. A., McCully C., 2014a, Astron Telegram, 6806, 1

Arcavi I., Valenti S., Hosseinzadeh G., Mccully C., Howell D. A., Sand D., 2014b, Astron Telegram, 6466, 1

Arcavi I., Valenti S., Howell D. A., Sand D., 2014c, Astron Telegram, 6304, 1

Baltay C. et al., 2013, PASP, 125, 683

Barbarino C. et al., 2015, MNRAS, 448, 2312

Barbon R., Ciatti, F., Rosino L., 1979, A\&A, 72, 287

Bayless A. J., Even W., Frey L. H., Fryer C. L., Roming P. W. a., Young P. a., 2015, ApJ, 805, 98

Bayless A. J. et al., 2013, ApJ, 764, L13

Becker A., 2015, ASCL, 04004B

Benetti S., Cappellaro E., Turatto M., della Valle M., Mazzali P. A., Gouiffes C., 1994, A\&A, 285, 147B

Bersten M. C., 2013, PhD thesis, 1, 168

Bertin E., 2010, ASCL, 10068B

Bertin E., Arnouts S., 1996, A\&AS, 117, 393

Blinnikov S. I., Bartunov O. S., 1993, A\&A, 273, 106

Bose S., Kumar B., 2014, ApJ, 782, 98

Bose S. et al., 2013, MNRAS, 433, 1871

Bose S. et al., 2015, MNRAS, 450, 2373

Breeveld A. A. et al., 2010, MNRAS, 406, 1687B

Brown P. J., Breeveld A. A., Holland S., Kuin P., Pritchard T., 2014, Astrophysics and Space Science, 354, 89

Brown P. J. et al., 2009, AJ, 137, 4517

Brown P. J. et al., 2010, ApJ, 721, 1608

Brown T. M. et al., 2013, PASP, 125, 1031

Chevalier R. A., 1976, ApJ, 207, 872

Chevalier R. A., Irwin C. M., 2011, ApJ, 729, L6

Childress M., Scalzo R., Yuan F., Schmidt B., Tucker B., 2013, Astron Telegram, 5455, 1

Conseil E. et al., 2013a, Central Bureau Electronic Telegrams, 3431,1

Conseil E., Klotz A., Coward D., Zadko J., Boer M., 2013b, Astron Telegram, 4849, 1

Dall'Ora M. et al., 2014, ApJ, 787, 139

de Vaucouleurs G., de Vaucouleurs A., Buta R., Ables H. D., Hewitt A. V., 1981, PASP, 93, 36

Dessart L. et al., 2008, ApJ, 675, 644

Dessart L., Hillier D. J., 2010, MNRAS, 410, no

Dessart L., Hillier D. J., Waldman R., Livne E., 2013, MNRAS, 433, 1745

Dhungana G. et al., 2015, preprint (arXiv.org, asroph:1509.01721)

Elias-Rosa N. et al., 2014, Astron Telegram, 6440, 1

Elias-Rosa N. et al., 2010, ApJ, 714, L254

Elias-Rosa N. et al., 2011, ApJ, 742, 6

Elmhamdi A. et al., 2003, MNRAS, 338, 939

Ergon M. et al., 2014, Astron Telegram, 5796, 1

Falco E. et al., 2014, Astron Telegram, 6721, 1

Falk S. W., Arnett W. D., 1973, ApJ, 180, L65

Faran T. et al., 2014a, MNRAS, 442, 844

Faran T. et al., 2014b, MNRAS, 445, 554

Ferrarese L. et al., 1996, ApJ, 464, 568

Filippenko A. V., 1982, PASP, 94, 715

Filippenko A. V., 1997, A\&A Rev., 35, 309

Filippenko A. V., Li W., Treffers R., Modjaz M., 2001, IAU Circ., 246,121

Firth R. E. et al., 2014, MNRAS, 446, 3895

Fisher A. K., 2000, PhD thesis, THE UNIVERSITY OF OKLAHOMA
Foreman-Mackey D., Hogg D. W., Lang D., Goodman J., 2013, PASP, 125,306

Fransson C., Chevalier R. A., 1989, ApJ, 343, 323

Fraser M. et al., 2011, MNRAS, 417, 1417

Fraser M. et al., 2014, MNRAS, 439, 5

Fraser M. et al., 2009, ApJ, 714, L280

Freedman W. L. et al., 2001, ApJ, 553, 47

Gal-Yam A. et al., 2011, ApJ, 736, 159

Galbany L. et al., 2015, 110

Gall E. E. E. et al., 2015, A\&A, 582, A3

Gandhi P. et al., 2013, ApJ, 767, 166

Gonzalez-Gaitan S. et al., 2015, MNRAS, 451, 2212

Hadjiyska E. et al., 2011, Astron Telegram, 3812, 1

Hamuy M., 2003, ApJ, 582, 905

Hamuy M., Suntzeff N. B., Gonzalez R., Martin G., 1988, AJ, 95, 63

Henden A. A., Levine S. E., Terrell D., Smith T. C., Welch D., 2012, JAVSO, 40, 430H

Holoien T. W.-S. et al., 2014, Astron Telegram, 6436, 1

Hsiao E. Y. et al., 2013, Astron Telegram, 5678, 1

Inserra C. et al., 2013, A\&A, 555, A142

Inserra C. et al., 2011, MNRAS, 417, 261

Inserra C. et al., 2012, MNRAS, 422, 1122

Itagaki K. et al., 2013, Central Bureau Electronic Telegrams, 3498, 1

Jenness T., Economou F., 2015, Astronomy and Computing, 9, 40

Jerkstrand A., Fransson C., Kozma C., 2011, A\&A, 530, A45

Jerkstrand a., Fransson C., Maguire K., Smartt S., Ergon M., Spyromilio J., 2012, A\&A, 546, A28

Jerkstrand, A., Smartt, S. J., Fraser, M., et al. 2014, MNRAS, 439, 3694

Jerkstrand a., Ergon M., Smartt S. J., Fransson C., Sollerman J., Taubenberger S., Bersten M., Spyromilio J., 2015a, A\&A, 573, A12

Jerkstrand a. et al., 2015b, MNRAS, 448, 2482

Jones M. I. et al., 2009, ApJ, 696, 1176

Kanbur S. M., Ngeow C., Nikolaev S., Tanvir N. R., Hendry M. A., 2003, A\&A, 411, 361

Kasen D., Woosley S. E., 2009, ApJ, 703, 2205

Kim, H.; Zheng W. F. A. V., 2015, Central Bureau Electronic Telegrams, 4168, 1

Kiyota S. et al., 2014, Astron Telegram, 6460, 1

Krisciunas K. et al., 2009, AJ, 137, 34

Leonard D. C. et al., 2002a, PASP, 114, 35

Leonard D. C. et al., 2002b, AJ, 124, 2490

Leonard D. C., Kanbur S. M., Ngeow C. C., Tanvir N. R., 2003, ApJ, 594, 247

Levesque E. M., Massey P., Olsen K. A. G., Plez B., Josselin E., Maeder A., Meynet G., 2005, ApJ, 628, 973

Li W. et al., 2011, MNRAS, 412, 1441

Li W. D., 2000, in AIP Conf. Proc., Vol. 522, AIP, pp. 103-106

Maguire K. et al., 2010, MNRAS, 404, 981

Maguire K. et al., 2012, MNRAS, 420, 3451

Maund J. R., Fraser M., Reilly E., Ergon M., Mattila S., 2015, MNRAS, 447, 3207

Maund J. R., Smartt S. J., Danziger I. J., 2005, MNRAS, 364, L33

Mazzali P. a. et al., 2007, ApJ, 670, 24

Mazzali P. a., Maurer I., Valenti S., Kotak R., Hunter D., 2010, MNRAS, 408, 87

Moriya T. J., Pruzhinskaya M. V., Ergon M., Blinnikov S. I., 2015, MNRAS, 455, 423

Morozova V., Piro A. L., Renzo M., Ott C. D., Clausen D., Couch S. M., Ellis J., Roberts L. F., 2015, ApJ, 814, 63

Morrell N., Phillips M. M., Contreras C., Marion G. H., Hsiao E. Y., Gall C., Stritzinger, D. M., Kirshner R. P., 2014, Central Bureau Electronic Telegrams, 3964, 2 
Mould J., Sakai S., 2008, ApJ, 686, L75

Munari U., Zwitter T., 1997, A\&A, 318, 269

Nakano S. et al., 2013, Central Bureau Electronic Telegrams, 3671, 1

Nakar E., Poznanski D., Katz B., 2015, eprint arXiv:1506.07185

Nakar E., Sari R., 2010, ApJ, 725, 904

Nicolas J. et al., 2014, Astron Telegram, 6714, 1

Nishimura K., 2014, Central Bureau Electronic Telegrams, 3964A

Ochner P., Tomasella L., Pastorello A., Benetti S., Cappellaro E., Turatto M., 2013, Astron Telegram, 5005, 1

Oke, J. B., Cohen, J. G., Carr, M., et al. 1995, PASP, 107, 375

Olivares F. et al., 2010, ApJ, 715, 833

Parker S., 2015, Central Bureau Electronic Telegrams, 4047, 1

Pastorello a., Aretxaga I., Zampieri L., Mucciarelli P., Benetti S., 2005, 1604-2004: Supernovae as Cosmological Lighthouses, 342,5

Pastorello a. et al., 2006, MNRAS, 370, 1752

Pastorello a. et al., 2009, MNRAS, 394, 2266

Pastorello a. et al., 2004, MNRAS, 347, 74

Patat F., Barbon R., Cappellaro E., Turatto M., 1994, A\&A, 282, 731

Pejcha O., Prieto J. L., 2015, ApJ, 806, 225

Popov D. V., 1993, ApJ, 414, 712

Poznanski D., 2013, MNRAS, 436, 3224

Poznanski D. et al., 2009, ApJ, 694, 1067

Poznanski D., Kostrzewa-Rutkowska Z., Wyrzykowski L., Blagorodnova N., 2015, MNRAS, 449, 1753

Poznanski D., Prochaska J. X., Bloom J. S., 2012, MNRAS, 426, 1465

Pozzo M. et al., 2006, MNRAS, 368, 1169

Pritchard T. A., Roming P. W. A., Brown P. J., Bayless A. J., Frey L. H., 2014, ApJ, 787, 157

Quimby R. M., Wheeler J. C., Hoflich P., Akerlof C. W., Brown P. J., Rykoff E. S., 2007, ApJ, 666, 1093

Rabinak I., Waxman E., 2011, ApJ, 728, 63

Rau A. et al., 2009, PASP, 121, 15

Rest A. et al., 2014, ApJ, 795, 44

Roming P. W. A. et al., 2005, Space Science Reviews, 120, 95

Roy R. et al., 2011, ApJ, 736, 76

Rubin ÃĆ. et al., 2015, eprint arXiv:1512.00733

Sanders N. E. et al., 2015, ApJ, 799, 208

Schlafly E. F., Finkbeiner D. P., 2011, ApJ, 737, 103

Schmidt B. P., Kirshner R. P., Eastman R. G., 1992, ApJ, 395, 366

Shappee B. J. et al., 2014, ApJ, 788, 48

Smartt S. J., 2009, A\&A Rev., 47, 63

Smartt S. J., 2015, Publications of the Astronomical Society of Australia, 32, e016

Smartt S. J., Eldridge J. J., Crockett R. M., Maund J. R., 2009, MNRAS, 395, 1409

Smartt S. J. et al., 2015, A\&A, 579, A40

Spiro S. et al., 2014, MNRAS, 439, 2873

Stanek K. Z. et al., 2014, Astron Telegram, 6301, 1S

Stetson P. B., 1987, PASP, 99, 191

Taddia F. et al., 2013, A\&A, 555, A10

Takats K. et al., 2015, MNRAS, 450, 18

Takáts K. et al., 2014, MNRAS, 438, 368

Takats K., Vinko J., 2006, MNRAS, 372, 1735

Takáts K., Vinkó J., 2012, MNRAS, 419, 2783

Taubenberger S. et al., 2009, MNRAS, 397, 677

Tomasella L. et al., 2015, Astron Telegram, 6906

Tomasella L. et al., 2014, Astronomische Nachrichten, 335, 841

Tomasella L. et al., 2013, MNRAS, 434, 1636

Turatto M., Benetti S., Cappellaro E., 2002, in From Twilight to Highlight: The Physics of Supernovae: Proceedings of the ESO/MPA/MPE Workshop Held at Garching, INAF, Osservatorio Astronomico di Padova, Vicolo dell'Osservatorio 5, 35122 Padova, Italia, p. 10
Valenti S. et al., 2008, MNRAS, 383, 1485

Valenti S. et al., 2014, MNRAS, 438, L101

Valenti S. et al., 2015, MNRAS, 448, 2608

Van Dyk S. D. et al., 2012, ApJ, 756, 131

Wang X., Wang L., Pain R., Zhou X., Li Z., 2006, ApJ, 645, 488

Waxman E., Meszaros P., Campana S., 2007, ApJ, 667, 351

Woosley S. E., Heger A., 2007, Physics Reports, 442, 269

Zwitter A., Munari A., Moretti A., 2004, IAU Circ., 8413, 1

\section{APPENDIX A: SAMPLE}

- SN 2013bu: SN 2013bu was discovered on 2013 Apr. 21.76 (UT dates are used throughout this paper) in NGC 7331 at J2000 coordinates (here and elsewhere) $\alpha=$ $22^{h} 37^{m} 05^{s} .60, \delta=+34^{\circ} 24^{\prime} 31.9^{\prime \prime}$ (Itagaki et al. 2013). It was classified on 2013 Apr. 24.08 as a young SN II a few days after the explosion (Ochner et al. 2013). Our followup observations started on 2013 Apr. 23.45 and continued with a few-day cadence until 2013 Sep. 1.24. The SN was not visible on 2013 Apr. 12.8 (Itagaki et al. 2013).

- SN 2013fs: SN 2013fs was discovered on 2013 Oct. 07.46 (Nakano et al. 2013) at $\alpha=23^{h} 19^{m} 44^{s} .67, \delta=$ $+10^{\circ} 11^{\prime} 04.5^{\prime \prime}$. It was classified on 2013 Oct. 9.2 as a young SN II (Childress et al. 2013). The classification spectrum exhibited narrow lines leading to a possible classification as an interacting Type IIn supernova (SN IIn). However, the narrow lines were visible only at early phases, placing SN 2013fs in the sample of SNe II that show signs of circumstellar material around the progenitor. A PTF detection of SN 2013fs (iPTF13dqy) on 2013 Oct. 6.24 and a nondetection the day before places the explosion epoch of SN 2013fs to be less than $24 \mathrm{hr}$ before the first detection (Yaron et al., in preparation). Our follow-up observations began on 2013 Oct. 7.27 and continued with several-day observing cadence until 2014 Jan. 22.06. Swift observations were also obtained in the month following discovery.

- LSQ13dpa: LSQ13dpa was discovered at coordinates $\alpha=11^{h} 01^{m} 12^{s} .91, \delta=-05^{\circ} 50^{\prime} 52.4^{\prime \prime}$ on 2013 Dec. 18.28 by the LSQ survey (Hadjiyska et al. 2011; Baltay et al. 2013) and classified on 2013 Dec. 20.3 as a young SN II (Hsiao et al. 2013). Our follow-up observations were initiated on 2013 Dec. 20.25 and continued with a few-day cadence for $\sim 200$ days. A nondetection 4 days prior to discovery confirms that LSQ13dpa was discovered within 4 days after its explosion.

- SN 2013ai: SN 2013ai was discovered at coordinates $\alpha=06^{h} 16^{m} 18^{s} .35, \delta=-21^{\circ} 22^{\prime} 32.9^{\prime \prime}$ on 2013 Mar. 1.66 (Conseil et al. 2013a). The object was classified by the Public ESO Spectroscopic Survey for Transient Objects (PESSTO; Smartt et al. 2015) as a young SN II (Conseil et al. 2013b). Our follow-up observations started on 2013 Mar. 5.06 and continued until the SN disappeared in the Sun's glare $\sim 83$ days after explosion.

- SN 2014cy: SN 2014cy was discovered on 2014 Aug. 31.0 at coordinates $\alpha=23^{h} 44^{m} 16^{s} .03, \delta=+10^{\circ} 46^{\prime} 12.5^{\prime \prime}$ (Nishimura 2014). Morrell et al. (2014) classified the object as a SN II around 10 days after explosion. However, a nondetection on 2013 Aug. 29.3 and a prediscovery LOSS detection on 2013 Aug. 31.3 place the explosion on Aug. 
30.3 , with an uncertainty of 1.0 day $^{12}$. LCOGT follow-up observations started on 2014 Sep. 3.39 and ended on 2015 Feb. 9.06. However, over the last month of monitoring the object was only marginally detected in the $i$ band.

- ASASSN-14ha: ASASSN-14ha was discovered by ASASSN on 2014 Sep. 10.29 at coordinates $\alpha=$ $04^{h} 20^{m} 01^{s} .41, \delta=-54^{\circ} 56^{\prime} 17.0^{\prime \prime}$ (Kiyota et al. 2014). We classified the object using the FLOYDS spectrograph as a young SN II on 2014 Sep. 13.68 (Arcavi et al. 2014b). LCOGT follow-up observations were initiated on 2014 Sep. 11.77 and continued with a few-day cadence until the end of Mar. 2015.

- ASASSN-14gm/SN 2014cx: ASASSN-14gm was discovered on 2014 Sep. 2.47 at coordinates $\alpha=$ $00^{h} 59^{m} 47^{s} .83, \delta=-07^{\circ} 34^{\prime} 19.3^{\prime \prime}$ (Holoien et al. 2014). Elias-Rosa et al. (2014) classified the object as a young SN II the day after discovery. At the same time (2014 Sep. 3.12) we began our LCOGT follow-up observations, which concluded in Mar. 2015.

- ASASSN-14dq: ASASSN-14dq was discovered on 2014 July 8.48 at coordinates $\alpha=21^{h} 57^{m} 59^{s} .97$, $\delta=+24^{\circ} 16^{\prime} 08.1^{\prime \prime}$. We classified this transient using the FLOYDS spectrograph on 2014 July 9.5 (Arcavi et al. 2014c) and observed it further with LCOGT facilities from 2014 July 10.74 to 2014 Dec. 30.07 .

- SN 2014dw: SN 2014dw was discovered at coordinates $\alpha=11^{h} 10^{m} 48^{s} .41, \delta=-37^{\circ} 27^{\prime} 02.2^{\prime \prime}$ on 2014 Nov. 6.589 (Parker 2015). We classified it as a SN II (Arcavi et al. 2014a). No recent prediscovery images are available. However, the classification spectrum was quite blue with conspicuous $\mathrm{H} \alpha$ lines, consistent with an object around a fortnight after explosion. We adopt JD $=2,456,958 \pm 10$ days as the explosion epoch.

- LSQ14gv: LSQ14gv was discovered at coordinates $\alpha=$ $10^{h} 54^{m} 11^{s} .71, \delta=-15^{\circ} 01^{\prime} 30.0^{\prime \prime}$ on 2014 Jan. 17.3 by the LSQ survey (Hadjiyska et al. 2011; Baltay et al. 2013) and classified by PESSTO on 2014 Jan. 23 as a young SN II (Ergon et al. 2014). Our follow-up observations started on 2014 Jan. 25.60 and continued for $\sim 3$ months. A nondetection on 2014 Jan. 13.17 places the explosion on 2014 Jan. $15.3 \pm 2$ days.

- ASASSN-14kg: ASASSN-14kg was discovered at coordinates $\alpha=01^{h} 44^{m} 38^{s} .38, \delta=+35^{\circ} 48^{\prime} 20.5^{\prime \prime}$ on 2014 Nov. 17.36 (Nicolas et al. 2014) and classified as a young SN II on 2014 Nov. 18.2 (Falco et al. 2014). Our follow-up observations started on 2014 Nov. 27.27, 10 days after discovery. However, the early rise and a nondetection on 2014 Nov. 11.38 suggest that the explosion epoch was soon after the nondetection. We followed the object until 2015 Mar. 7.09 when the SN disappeared in the glare of the Sun.

- SN 2015W: SN 2015W was discovered by LOSS on 2015 Jan. 12.17 at coordinates $\alpha=06^{h} 57^{m} 43^{s} .03, \delta=$ $+13^{\circ} 34^{\prime} 45.7^{\prime \prime}$ and was reported to the TOCP pages of the CBAT. It was classified as an SN II $\sim 10$ days after explosion (Tomasella et al. 2015). Our follow-up observations started on 2015 Jan. 12.92 and continued for four months until the object disappeared in the Sun's glare.

12 KAIT nondetection and prediscovery detection reported on the TOCP of the CBAT

\section{APPENDIX B: LCOGTSNPIPE}

In this section we will briefly describe the lcogtsnpipe pipeline used to ingest and automatically reduce the photometric data presented in this paper and all the data collected under the LCOGT Supernova Key Project ("The next-generation sample of supernovae"; PI D. A. Howell). It is a PYTHON module based on a few main Python packages (numpy, MySQLdb, PyRAF, pyfits, matplotlib) and uses other astronomical software at different stages of the reduction. In particular, lcogtsnpipe uses HOTPANTS (Becker 2015), SEXTRACTOR (Bertin \& Arnouts 1996), SWARP (Bertin 2010), and astro-scrappy ${ }^{13}$. The instrumental magnitudes are computed using DAOPHOT (Stetson 1987) and the PSF subtraction technique. We use the IRAF implementation developed by Enrico Cappellaro in the snoopy package ${ }^{14}$. The lcogtsnpipe "stages" are described in detail below.

- Ingestion: The LCOGT network is a world-wide facility that is able to observe the full sky nearly continuously (Brown et al. 2013). At the end of the night for each LCOGT site, the raw data are preprocessed using the lcogtorac pipeline (Brown et al. 2013; Jenness \& Economou 2015). The prereduced data are then sent to the IPAC archive, where LCOGT users can access them. The ingestion stage is triggered within $1 \mathrm{hr}$ after the end of the night for each site. The images are stored locally on a dedicated machine where they are processed. The key header information is stored in a database to allow lcogtsnpipe to identify the new frames by telescope, filter, instrument, object name or coordinates.

- Astrometry: The ORAC LCOGT prereduction produces an astrometric solution for each frame. However, this fails on a subset of images, so lcogtsnpipe runs a custom astrometry routine to solve these frames. Starting from an initial guess based on the telescope pointing and the image pixel scale, it computes a cross-match of extracted sources in the frame with available all-sky catalogs (2MASS, UCAC).

- Point-spread function: The next stage of lcogtsnpipe is to compute the PSF of each frame. This is done using DAOPHOT within PyRAF. It also creates a FITS table with a catalog of $5 \sigma$ detections and their aperture and PSF instrumental magnitudes. This table will be used to compute the absolute calibration in the next stage of the pipeline.

- Instrumental PSF photometry: This stage uses DAOPHOT within PyRAF to fit the SN. At the same time, a low-order polynomial fit to the background is computed to remove the host-galaxy contamination. This implementation was developed by E. Cappellaro for the snoopy package.

- Zeropoint and colour-term calibration: The pipeline cross-matches the FITS table produced in the PSF stage with a local catalog of magnitudes. Since we are mainly observing in $B V$ gri, our first choice is the APASS catalog (Henden et al. 2012). However, lcogtsnpipe is also able to calibrate using SDSS, Landolt fields, or the natural LCOGT system. Several options are available to compute the zeropoint and colour terms. The default option is to use fixed

13 https://github.com/cmccully/astroscrappy

14 http://sngroup.oapd.inaf.it/snoopy.html 
colour terms that have been measured during the commissioning of the LCOGT telescopes (see Table B1).

- Apparent magnitudes of the SN: Once the instrumental magnitudes and zeropoints have been computed for each night, this stage computes the apparent magnitude of the SN using first-order colour corrections. Our apparent magnitudes are usually calibrated to the Landolt photometric system (Vega magnitudes) or to the Sloan Photometric System (AB magnitudes).

- Difference imaging: This stage is applied when the $\mathrm{SN}$ is in crowded regions where a background estimate is complicated. We use HOTPANTS to compute the convolution kernel after the target and the reference images are aligned (with the gregister task in PyRAF). The previous steps to compute the apparent magnitudes can be applied to the difference images.

Several other stages are available within lcogtsnpipe that can be run automatically or in interactive mode to improve our photometric reduction, reject bad-quality data, and check each step of the reduction. These stages are usually run manually on each object as a sanity check, to ensure that the photometric product is ready for publication. At each stage the database is updated. Once the information is stored in the database, it is available to all members of the Key Project through the Supernova Exchange (SNEx; Arcavi et al., in preparation), usually within $24 \mathrm{hr}$ of observation.

\section{APPENDIX C: NEBULAR SPECTRA REDUCTION}

At Keck, we obtained one or two 1200-s exposures, and reduced the spectrum using routines written specifically for LRIS in the Carnegie Python (CARPY) package. The twodimensional (2D) images were flat-fielded, corrected for distortion along the $y$ (slit) axis, wavelength calibrated with comparison-lamp spectra, and cleaned of cosmic rays before extracting the 1D spectrum of the target. This spectrum was flux calibrated using a sensitivity function derived from a standard star obtained the same night with the same instrument configuration. The standard-star spectrum was also used to remove the telluric sky absorption features.

The Gemini spectra were reduced using the custom pipeline developed by C. McCully ${ }^{15}$.

Details of the spectra can be found in Table C1.
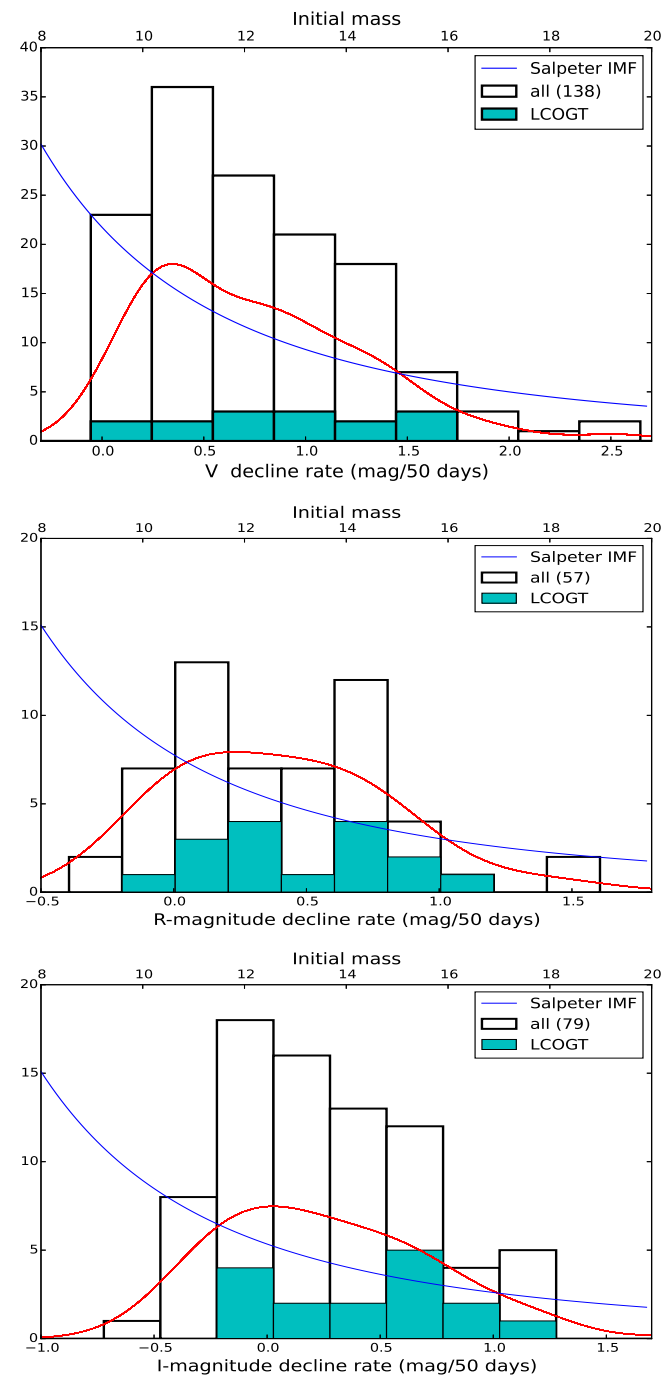

Figure D1. $s_{50}$ distributions for a sample of SNe II in $V$ (top panel), $R$ (central panel), and $I$ (lower panel). In blue we plot a Salpeter initial mass function to emphasize what the declinerate distribution would look like if more-massive progenitors are related to faster decline rate SNe. In red is the kernel density estimate using a Gaussian kernel for the same set of data.

\section{APPENDIX D: FIGURES AND TABLES}

15 https://github.com/cmccully/lcogtgemini 
Table B1. LCOGT Colour Terms, Zeropoints, and Extinction Coefficients

\begin{tabular}{|c|c|c|c|c|c|c|}
\hline Filter $^{a}$ & Colour & $\begin{array}{c}\text { Zeropoint } \\
2013-2014-2015\end{array}$ & Colour Term & Extinction & Telescope & Instrument $b$ \\
\hline$g$ & $g r$ & $23.05-22.90-22.75$ & $0.137(021)$ & $0.21(04)$ & $1 \mathrm{~m}-03$ & SBIG \\
\hline$r$ & $r i$ & $22.80-22.65-22.50$ & $-0.005(011)$ & $0.10(04)$ & $1 \mathrm{~m}-03$ & SBIG \\
\hline$i$ & ri & $22.10-22.00-21.90$ & $0.007(014)$ & $0.07(04)$ & $1 \mathrm{~m}-03$ & SBIG \\
\hline$B$ & $B V$ & $22.44-22.30-22.15$ & $-0.025(022)$ & $0.20(06)$ & $1 \mathrm{~m}-03$ & SBIG \\
\hline$V$ & $B V$ & $22.80-22.63-22.50$ & $0.017(025)$ & $0.14(07)$ & $1 \mathrm{~m}-03$ & SBIG \\
\hline$g$ & $g r$ & $---23.60-23.50$ & $0.109(014)$ & $0.14(03)$ & $1 \mathrm{~m}-04$ & Sinistro \\
\hline$r$ & $r i$ & $---23.50-23.35$ & $0.027(013)$ & $0.08(02)$ & $1 \mathrm{~m}-04$ & Sinistro \\
\hline$i$ & $r i$ & $---23.10-22.90$ & $0.036(016)$ & $0.06(03)$ & $1 \mathrm{~m}-04$ & Sinistro \\
\hline$B$ & $B V$ & $---23.00-22.85$ & $-0.024(011)$ & $0.23(03)$ & $1 \mathrm{~m}-04$ & Sinistro \\
\hline$V$ & $B V$ & $---23.25-23.15$ & $-0.014(030)$ & $0.12(05)$ & $1 \mathrm{~m}-04$ & Sinistro \\
\hline$g$ & $g r$ & $23.05-22.85-22.70$ & $0.120(018)$ & $0.14(03)$ & $1 \mathrm{~m}-05$ & SBIG \\
\hline$r$ & $r i$ & $22.80-22.65-22.50$ & $-0.002(018)$ & $0.08(02)$ & $1 \mathrm{~m}-05$ & SBIG \\
\hline$i$ & $r i$ & $22.15-21.00-21.90$ & $0.019(022)$ & $0.06(03)$ & $1 \mathrm{~m}-05$ & SBIG \\
\hline$B$ & $B V$ & $22.45-22.35-22.15$ & $-0.035(019)$ & $0.23(03)$ & $1 \mathrm{~m}-05$ & SBIG \\
\hline$V$ & $B V$ & $22.75-22.60-22.45$ & $0.0(036)$ & $0.12(05)$ & $1 \mathrm{~m}-05$ & SBIG \\
\hline$g$ & $g r$ & $22.95-22.75-22.60$ & $0.114(018)$ & $0.15(07)$ & $1 \mathrm{~m}-08$ & SBIG \\
\hline$r$ & $r i$ & $22.80-22.60-22.40$ & $-0.004(017)$ & $0.09(04)$ & $1 \mathrm{~m}-08$ & SBIG \\
\hline$i$ & $r i$ & $22.05-21.95-21.70$ & $0.024(023)$ & $0.07(04)$ & $1 \mathrm{~m}-08$ & SBIG \\
\hline$B$ & $B V$ & $22.25-22.10-22.00$ & $-0.039(022)$ & $0.22(05)$ & $1 \mathrm{~m}-08$ & SBIG \\
\hline$V$ & $B V$ & $22.65-22.45-22.30$ & $-0.005(035)$ & $0.15(05)$ & $1 \mathrm{~m}-08$ & SBIG \\
\hline$g$ & $g r$ & $---23.45-23.35$ & $0.109(014)$ & $0.14(03)$ & $1 \mathrm{~m}-09$ & Sinistro \\
\hline$r$ & $r i$ & $---23.40-23.30$ & $0.027(013)$ & $0.08(02)$ & $1 \mathrm{~m}-09$ & Sinistro \\
\hline$i$ & $r i$ & $---23.10-23.00$ & $0.036(016)$ & $0.06(03)$ & $1 \mathrm{~m}-09$ & Sinistro \\
\hline$B$ & $B V$ & $---22.75-22.60$ & $-0.024(011)$ & $0.23(03)$ & $1 \mathrm{~m}-09$ & Sinistro \\
\hline$V$ & $B V$ & $---23.15-23.05$ & $-0.014(030)$ & $0.12(05)$ & $1 \mathrm{~m}-09$ & Sinistro \\
\hline$g$ & $g r$ & $23.00-22.85-22.70$ & $0.112(016)$ & $0.16(05)$ & $1 \mathrm{~m}-10$ & SBIG \\
\hline$r$ & ri & $22.75-22.60-22.50$ & $-0.001(014)$ & $0.10(04)$ & $1 \mathrm{~m}-10$ & SBIG \\
\hline$i$ & $r i$ & $22.10-22.05-21.95$ & $0.013(022)$ & $0.07(04)$ & $1 \mathrm{~m}-10$ & SBIG \\
\hline$B$ & $B V$ & $22.35-22.20-22.05$ & $-0.030(020)$ & $0.19(09)$ & $1 \mathrm{~m}-10$ & SBIG \\
\hline$V$ & $B V$ & $22.60-22.50-22.35$ & $-0.019(025)$ & $0.14(06)$ & $1 \mathrm{~m}-10$ & SBIG \\
\hline$g$ & $g r$ & $23.05-22.90-22.75$ & $0.137(021)$ & $0.21(04)$ & $1 \mathrm{~m}-11$ & SBIG \\
\hline$r$ & $r i$ & $22.80-22.65-22.50$ & $-0.005(011)$ & $0.10(04)$ & $1 \mathrm{~m}-11$ & SBIG \\
\hline$i$ & $r i$ & $22.10-22.00-21.90$ & $0.007(014)$ & $0.07(04)$ & $1 \mathrm{~m}-11$ & SBIG \\
\hline$B$ & $B V$ & $22.44-22.30-22.15$ & $-0.025(022)$ & $0.20(06)$ & $1 \mathrm{~m}-11$ & SBIG \\
\hline$V$ & $B V$ & $22.80-22.63-22.50$ & $0.017(025)$ & $0.14(07)$ & $1 \mathrm{~m}-11$ & SBIG \\
\hline$g$ & $g r$ & $23.05-22.90-22.75$ & $0.112(016)$ & $0.16(05)$ & $1 \mathrm{~m}-12$ & SBIG \\
\hline$r$ & ri & $22.80-22.65-22.50$ & $-0.001(014)$ & $0.10(04)$ & $1 \mathrm{~m}-12$ & SBIG \\
\hline$i$ & $r i$ & $22.10-22.00-21.90$ & $0.013(022)$ & $0.07(04)$ & $1 \mathrm{~m}-12$ & SBIG \\
\hline$B$ & $B V$ & $22.44-22.30-22.15$ & $-0.030(020)$ & $0.19(09)$ & $1 \mathrm{~m}-12$ & SBIG \\
\hline$V$ & $B V$ & $22.80-22.63-22.50$ & $-0.019(025)$ & $0.14(06)$ & $1 \mathrm{~m}-12$ & SBIG \\
\hline$g$ & $g r$ & $23.05-22.90-22.75$ & $0.112(016)$ & $0.16(05)$ & $1 \mathrm{~m}-13$ & SBIG \\
\hline$r$ & $r i$ & $22.80-22.65-22.50$ & $-0.001(014)$ & $0.10(04)$ & $1 \mathrm{~m}-13$ & SBIG \\
\hline$i$ & $r i$ & $22.10-22.00-21.90$ & $0.013(022)$ & $0.07(04)$ & $1 \mathrm{~m}-13$ & SBIG \\
\hline$B$ & $B V$ & $22.44-22.30-22.15$ & $-0.030(020)$ & $0.19(09)$ & $1 \mathrm{~m}-13$ & SBIG \\
\hline$V$ & $B V$ & $22.80-22.63-22.50$ & $-0.019(025)$ & $0.14(06)$ & $1 \mathrm{~m}-13$ & SBIG \\
\hline
\end{tabular}

a Zeropoint, colour term, and extinction coefficient have been measured during photometric nights using the following equation: $m_{i}-m_{c}=Z+C_{i} \times$ colour $+K \times$ airmass $i$, where $m_{i}$ is the instrumental magnitude for an observed star, $m_{c}$ is the magnitude from the APASS catalog, $C_{i}$ is the colour of this star from the APASS catalog, and airmass $i$ is the airmass at which the star was observed.

$b$ 1m0-08 (McDonald Observatory, USA); 1m0-10, 1m0-12, 1m0-13 (Sutherland, South Africa), 1m0-04, 1m0-05, 1m0-09 (Cerro Tololo, Chile); 1m0-03, 1m0-11 (Siding Spring, Australia). 
Table C1. Nebular spectra of SNe type II

\begin{tabular}{|c|c|c|c|c|c|c|c|}
\hline Object & Setup & Exposure & Epoch & Phase & $\mathrm{PI}$ & Reducer & Observer $^{a}$ \\
\hline ASASSN-14dq & Keck+LRIS (uvir 1.0") & $1 \times 1200 \mathrm{~s}$ & 2015 June 16.47 & 350 & Filippenko & M.G. & M.G, A.F., W.Z. \\
\hline ASASSN-14dq & Keck+LRIS (uvir $1.0^{\prime \prime}$ ) & $1 \times 1200 \mathrm{~s}$ & 2015 Oct. 10.34 & 464 & Filippenko & M.G. & M.G. \\
\hline ASASSN-14gm & Keck+LRIS (uvir $1.0^{\prime \prime}$ ) & $1 \times 1200 \mathrm{~s}$ & 2015 Oct. 10.39 & 405 & Filippenko & M.G. & M.G. \\
\hline SN $2015 \mathrm{~W}$ & Keck+LRIS (uvir 1.0") & $2 \times 1200 \mathrm{~s}$ & 2015 Oct. 10.60 & 281 & Filippenko & M.G. & M.G. \\
\hline ASASSN-14gm & Gemini+GMOS $\left(B 600+\mathrm{R} 400 \quad 1.5^{\prime \prime}\right)$ & $2 \times 900 \mathrm{~s}$ & 2015 July 27.62 & 330 & Howell & C. Mc. & Service \\
\hline ASASSN-14dq & Gemini+GMOS (B600+R400 $\left.1.0^{\prime \prime}{ }^{\prime}\right)$ & $2 \times 1900 \mathrm{~s}$ & 2015 Oct. 09 & 463 & Valenti & S.V. & Service \\
\hline SN $2015 \mathrm{~W}$ & Gemini-GMOS (R400 $\left.1.0^{\prime \prime}\right)$ & $2 \times 1900 \mathrm{~s}$ & 2015 Dec. 07 & 339 & Valenti & S.V. & Service \\
\hline
\end{tabular}

$a$ M.L.G. = M. L. Graham; A.F. = A. Filippenko; C.Mc. = C. McCully; W.Z. = W. Zheng; S.V. = S. Valenti

Table D1. Photometric Data (Complete table available in the online version of the paper)

\begin{tabular}{|c|c|c|c|c|c|c|c|c|c|}
\hline Date & JD & $\mathrm{mag}^{a}$ & Filter & telescope $^{b}$ & Date & JD & mag & Filter & telescope \\
\hline $\mathrm{xx}$ & 2456407.372 & $\begin{array}{l}12.7630 .069 \\
10\end{array}$ & UW2 & UVOT & $\mathrm{x}$ & 2456407.374 & $\begin{array}{lll}13.711 & 0.043\end{array}$ & $\mathrm{~V}$ & UVOT \\
\hline
\end{tabular}

a (a) Data have not been corrected for extinction; (b) UVOT (Swift Telescope); CSP (Cerro Tololo, Chile); 1m0-08 (McDonald Observatory, USA); 1m0-10, 1m0-12, 1m0-13 (Sutherland, South Africa), 1m0-04, 1m005, 1m0-09 (Cerro Tololo, Chile); 1m0-03, 1m0-11 (Siding Spring, Australia). 

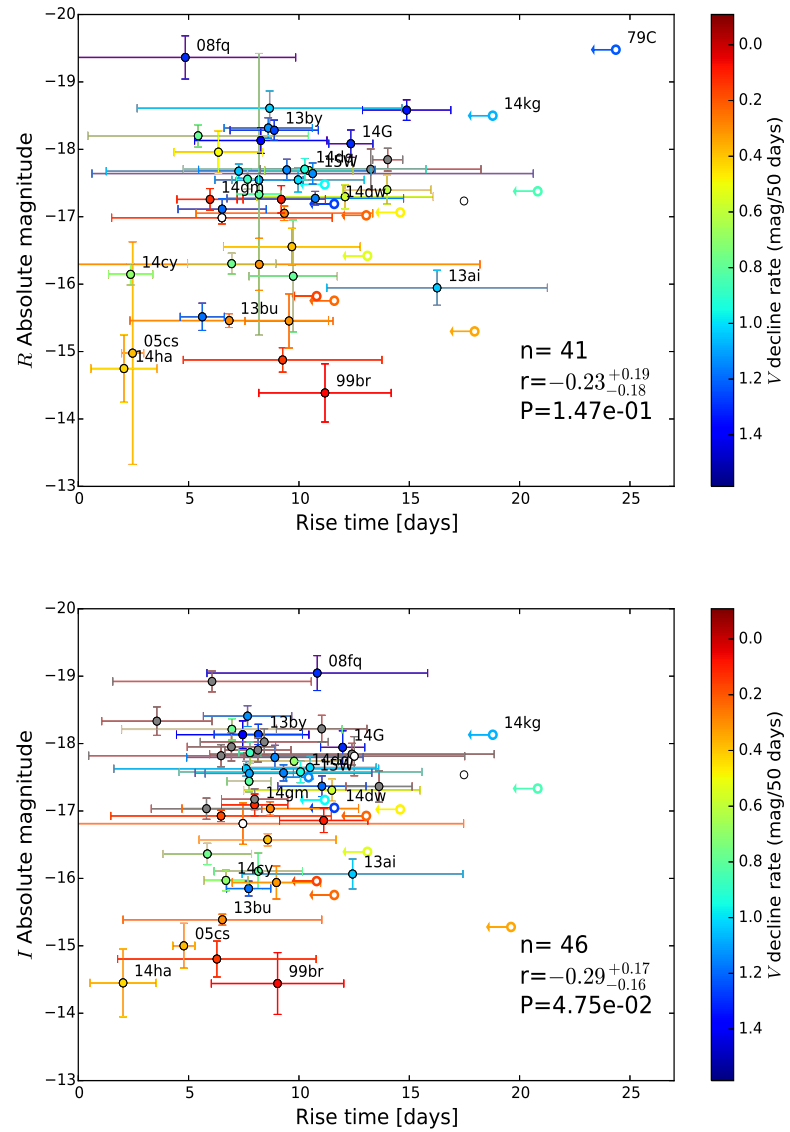

Figure D2. Absolute magnitude as a function of rise time for a sample of SNe II. Objects are colour-coded with the $V$-band decline rate $s_{50 \mathrm{~V}}$. Top panel: $R$ band. Bottom panel: $I$ band. Data from Poznanski et al. (2015) have also been added in the $I$ band.
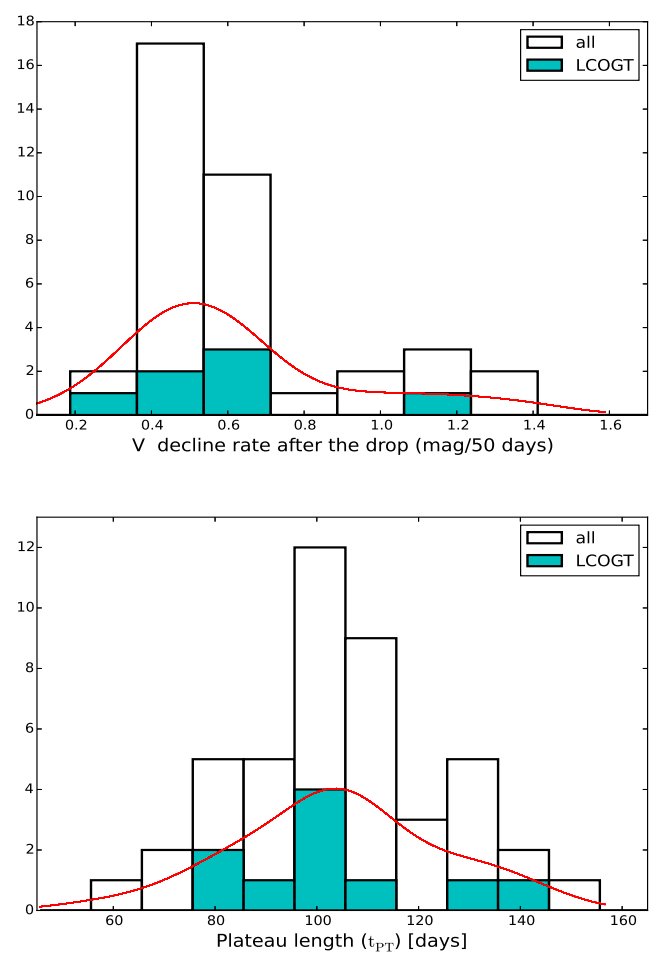

Figure D3. Top panel: Distribution of light-curve decline rates $s_{50 V}$ after the drop for a sample of SNe II in $V$. Bottom panel: Distribution of plateau length $t_{\mathrm{PT}}$ for a sample of SNe II in $V$. In red is the kernel density estimate using a Gaussian kernel for the same set of data.

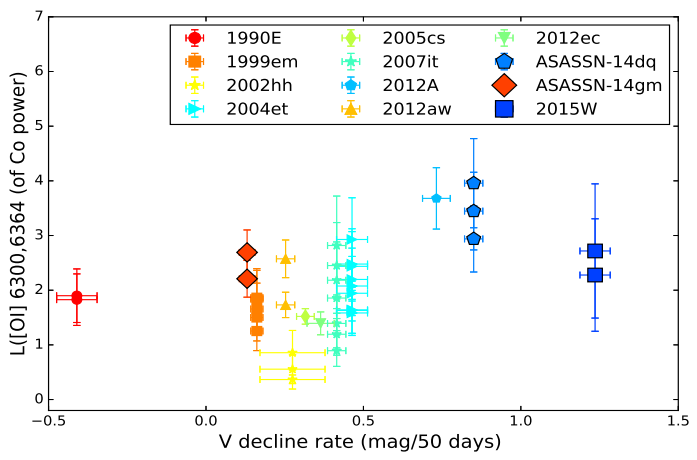

Figure D4. The [O I] $\lambda \lambda 6300,6364$ luminosity (normalized to the ${ }^{56}$ Co decay power) for the Jerkstrand et al. (2015b) sample of SNe and three objects from our sample as a function of $\mathrm{V}$ band decline rate. The [O I] $\lambda \lambda 6300,6364$ luminosities for all epochs between 200 and 500 days after explosion are plotted and are visible as multiple-points for each object. 
Table D2: Slope Data

\begin{tabular}{|c|c|c|c|c|c|c|c|c|c|c|c|c|c|c|c|c|c|c|}
\hline $\mathrm{SN}$ & $M(V) * \Delta M$ & $\begin{array}{c}850(\mathrm{~V}) \\
(\mathrm{V})\end{array}$ & $\Delta \mathrm{s} 50(\mathrm{~V})$ & ph_start & $\begin{array}{c}p^{p h} \text { stop } \\
(V)\end{array}$ & $\begin{array}{c}M(R) \\
(V)\end{array}$ & $\Delta M$ & $\begin{array}{c}s 50(R) \\
(R)\end{array}$ & $\Delta s 50(R)$ & ph_start & $\begin{array}{c}p h \text { stop } \\
(R) \\
(R)\end{array}$ & $\begin{array}{c}M(I) \\
(R) \\
\end{array}$ & $\Delta M$ & $\begin{array}{c}850(I) \\
(I) \\
\end{array}$ & $\Delta s 50(I)$ & ph_start & $\begin{array}{c}p^{p h}{ }_{(I)}^{\text {stop }} \\
(I)\end{array}$ & \\
\hline $\begin{array}{l}2013 \mathrm{ai} \\
2013 \mathrm{buu}\end{array}$ & $\begin{array}{l}16.80 \\
16.33\end{array}$ & $\begin{array}{l}0.02 \\
0.04\end{array}$ & $\begin{array}{l}0.02099 \\
0.0053\end{array}$ & $\begin{array}{l}0.0006 \\
0.0013\end{array}$ & $\begin{array}{l}17.6 \\
6.2\end{array}$ & $\begin{array}{l}54.5 \\
48.1\end{array}$ & $\begin{array}{l}16.59 \\
15.61\end{array}$ & $\begin{array}{l}0.02 \\
0.02\end{array}$ & $\begin{array}{l}0.0129 \\
0.0014\end{array}$ & $\begin{array}{l}0.0004 \\
0.0005\end{array}$ & $\begin{array}{l}17.6 \\
6.2\end{array}$ & $\begin{array}{l}64.5 \\
57.1\end{array}$ & $\begin{array}{l}16.29 \\
15.63\end{array}$ & $\begin{array}{l}0.05 \\
0.01\end{array}$ & $\begin{array}{l}0.0112 \\
-0.0027\end{array}$ & $\begin{array}{l}0.0009 \\
0.0004\end{array}$ & $\begin{array}{l}19.6 \\
6.2\end{array}$ & $\begin{array}{l}63.5 \\
55.1\end{array}$ \\
\hline $2013 \mathrm{fs}$ & $\begin{array}{l}16.03 \\
16.03\end{array}$ & 0.04 & 0.0158 & 0.0014 & 4.5 & 58.4 & $\begin{array}{l}15.01 \\
15.98\end{array}$ & 0.03 & 0.0075 & 0.0009 & 14.5 & 60.4 & $\begin{array}{l}10.00 \\
15.80\end{array}$ & $\begin{array}{l}0.01 \\
0.03\end{array}$ & 0.0065 & 0.0008 & $\begin{array}{l}0.2 \\
14.5\end{array}$ & 69.6 \\
\hline
\end{tabular}

Table D3: Bolometric light curve parameters

\begin{tabular}{|c|c|c|c|c|c|c|c|c|c|c|c|c|}
\hline SN & $\log 10(\operatorname{Lum} 1(0))$ & $S 1$ & $\Delta S 1$ & $\begin{array}{c}p h \_ \text {start } \\
\text { [days] }\end{array}$ & $\begin{array}{c}\text { ph_stop } \\
\text { [days] }\end{array}$ & $\log 10(\operatorname{Lum} 2(0))$ & $S 2$ & $\Delta S 2$ & $\begin{array}{c}\text { ph_start } \\
\text { [days] }\end{array}$ & $\begin{array}{c}\text { ph_stop } \\
\text { [days] }\end{array}$ & $\begin{array}{c}\mathrm{Ni} \\
\mathrm{M}_{\odot}\end{array}$ & $\begin{array}{c}\Delta N i \\
\mathrm{M}_{\odot}\end{array}$ \\
\hline 2013ai & 41.62 & -0.0081 & 0.0001 & 18.6 & 49.5 & 41.47 & -0.0057 & 0.0005 & 54.5 & 92.2 & - & - \\
\hline 2013bu & 41.24 & -0.0044 & 0.0003 & 6.2 & 35.1 & 41.18 & -0.0020 & 0.0003 & 35.1 & 78.1 & 0.0021 & 0.0007 \\
\hline $2013 \mathrm{fs}$ & 42.47 & -0.0146 & 0.0009 & 4.5 & 27.6 & 42.19 & -0.0054 & 0.0002 & 31.5 & 75.5 & 0.0545 & 0.0003 \\
\hline - & - & - & - & - & - & - & - & - & - & - & - & - \\
\hline
\end{tabular}

${ }^{*}$ the slope is computed with the following equation: $\log 10(\operatorname{Lum}(\mathrm{t}))=\log 10(\operatorname{Lum}(0))+\mathrm{S} 1 \times \mathrm{t}$

Table D4: Bolometric MCMC light curve parameters

\begin{tabular}{ccccccccccccc}
\hline SN & $T_{p t}$ & $\Delta T_{p t}$ & $A 0$ & $A 0+$ & $A 0-$ & $W 0$ & $W 0+$ & $W 0-$ & $M 0$ & $M 0+$ & $M 0-$ & P0 \\
\hline 2013bu & 102.8 & 4.5 & -1.14330 & 0.00008 & 0.00011 & 3.85663 & 0.00011 & 0.00011 & 39.73643 & 0.00011 & 0.00010 & -0.0038 \\
2013fs & 86.2 & 0.5 & -0.60601 & 0.00010 & 0.00009 & 4.85452 & 0.00010 & 0.00010 & 41.17148 & 0.00010 & 0.00009 & -0.0038 \\
LSQ13dpa & 129.9 & 2.0 & -0.52529 & 0.00010 & 0.00009 & 4.60926 & 0.00010 & 0.00010 & 41.16555 & 0.00007 & 0.00010 & -0.0038 \\
- & - & - & - & - & - & - & - & - & - & - & - & - \\
\hline
\end{tabular}

Table D5: MCMC parameters on V band light curves

\begin{tabular}{ccccccccccccc}
\hline SN & $t_{p t}$ & $\Delta t_{p t}$ & $a 0$ & $a 0+$ & $a 0-$ & $w 0$ & $w 0+$ & $w 0-$ & $m 0$ & $m 0+$ & $m 0-$ & $\mathrm{p} 0$ \\
\hline 2013bu & 103.1 & 4.5 & 3.08170 & 0.15800 & 0.14293 & 3.51516 & 0.60600 & 0.60607 & 20.27520 & 0.14960 & 0.13669 & 0.0120 \\
$2013 \mathrm{fs}$ & 82.7 & 0.5 & 1.59863 & 0.09900 & 0.07214 & 2.27769 & 2.47320 & 2.47329 & 18.76480 & 0.13160 & 0.08128 & 0.0120 \\
$2008 \mathrm{M}$ & 85.7 & 9.0 & 2.05115 & 0.03200 & 0.03193 & 2.67883 & 0.39460 & 0.39467 & 18.95140 & 0.02770 & 0.02715 & 0.0116 \\
- & - & - & - & - & - & - & - & - & - & - & - & - \\
\hline
\end{tabular}

